\title{
Decentralized Particle Filter with Arbitrary State Decomposition
}

\author{
Tianshi Chen, Member, IEEE, Thomas B. Schön, Member, IEEE, Henrik Ohlsson, Member, IEEE, \\ and Lennart Ljung, Fellow, IEEE
}

\begin{abstract}
In this paper, a new particle filter (PF) which we refer to as the decentralized PF (DPF) is proposed. By first decomposing the state into two parts, the DPF splits the filtering problem into two nested sub-problems and then handles the two nested sub-problems using PFs. The DPF has the advantage over the regular PF that the DPF can increase the level of parallelism of the PF. In particular, part of the resampling in the DPF bears a parallel structure and can thus be implemented in parallel. The parallel structure of the DPF is created by decomposing the state space, differing from the parallel structure of the distributed PFs which is created by dividing the sample space. This difference results in a couple of unique features of the DPF in contrast with the existing distributed PFs. Simulation results of two examples indicate that the DPF has a potential to achieve in a shorter execution time the same level of performance as the regular PF.
\end{abstract}

Index Terms-Particle filtering, parallel algorithms, nonlinear system, state estimation.

\section{INTRODUCTION}

In this paper, we study the filtering problem for the following nonlinear discrete-time system

$$
\begin{aligned}
\xi_{t+1} & =f_{t}\left(\xi_{t}, v_{t}\right) \\
y_{t} & =h_{t}\left(\xi_{t}, e_{t}\right)
\end{aligned}
$$

where $t$ is the discrete-time index, $\xi_{t} \in \mathbb{R}^{n_{\xi}}$ is the state at time $t, y_{t} \in \mathbb{R}^{n_{y}}$ is the measurement output, $v_{t} \in \mathbb{R}^{n_{v}}$ and $e_{t} \in \mathbb{R}^{n_{e}}$ are independent noises whose known distributions are independent of $t, \xi_{t}$ and $y_{t}$, and $f_{t}(\cdot)$ and $h_{t}(\cdot)$ are known functions. The filtering problem consists of recursively estimating the posterior density $p\left(\xi_{t} \mid y_{0: t}\right)$ where, $y_{0: t} \triangleq\left\{y_{0}, \ldots, y_{t}\right\}$. Analytic solutions to the filtering problem are only available for a relatively small and restricted class of systems, the most important being the Kalman filter [19] which assumes that system (1) has a linear-Gaussian structure. A class of powerful numerical algorithms to the filtering problem are particle filters (PFs), which are sequential Monte Carlo methods based on particle representations of probability densities [2]. Since the seminal work [15], PFs have become an important tool in handling the nonlinear non-Gaussian filtering problem, and have found many applications in statistical signal processing, economics and engineering; see e.g., $[2,12,14,16]$ for recent surveys of PFs.

Copyright (c) 2010 IEEE. Personal use of this material is permitted. However, permission to use this material for any other purposes must be obtained from the IEEE by sending a request to pubs-permissions@ieee.org.

The authors are with the Division of Automatic Control, Department of Electrical Engineering, Linköping University, Linköping, 58183, Sweden. Email: \{tschen, schon, ohlsson, ljung\}@ @isy.liu.se. Phone: +46 (0)13 282226. Fax: +46 (0)13282622.
In this paper, a new PF, which we refer to as the decentralized PF (DPF), will be proposed. By first decomposing the state into two parts, the DPF splits the filtering problem of system (1) into two nested sub-problems and then handles the two nested sub-problems using PFs. The DPF has the advantage over the regular PF that the DPF can increase the level of parallelism of the PF in the sense that besides the particle generation and the importance weights calculation, part of the resampling in the DPF can also be implemented in parallel. As will be seen from the DPF algorithm, there are actually two resampling steps in the DPF. The first resampling in the DPF, like the resampling in the regular PF, cannot be implemented in parallel, but the second resampling bears a parallel structure and can thus be implemented in parallel. Hence, the parallel implementation of the DPF can be used to shorten the execution time of the PF.

As pointed out in [5], the application of PFs in real-time systems is limited due to its computational complexity which is mainly caused by the resampling involved in the PF. The resampling is essential in the implementation of the $\mathrm{PF}$ as without resampling the variance of the importance weights will increase over time [13]. The resampling however introduces a practical problem. The resampling limits the opportunity to parallelize since all the particles must be combined, although the particle generation and the importance weights calculation of the PF can still be realized in parallel [13]. Therefore, the resampling becomes a bottleneck to shorten the execution time of the PF. Recently, some distributed resampling algorithms for parallel implementation of PFs have been proposed in $[5,22]$. The idea of the distributed resampling is to divide the sample space into several strata or groups such that the resampling can be performed independently for each stratum or group and can thus be implemented in parallel. The effect of different distributed resampling algorithms on the variance of the importance weights has been analyzed in [22]. Based on the introduced distributed resampling algorithms, a couple of distributed PFs have been further proposed in [5,22], such as the distributed resampling with proportional allocation PF (DRPA-PF) and the distributed resampling with nonproportional allocation PF (DRNA-PF).

The underlying idea of the DPF is different from that of the existing distributed PFs, while they all have parallel structure. The parallel structure of the DPF is created by decomposing the state space, differing from the parallel structure of the distributed PFs which is created by dividing the sample space. This difference results in a couple of unique features of the DPF in contrast with the existing distributed PFs. First, 

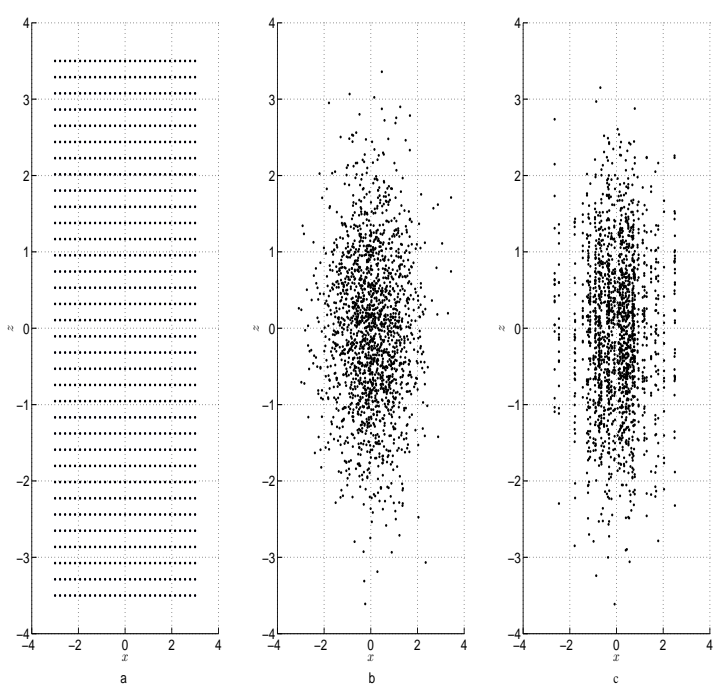

Fig. 1. Patterns for points where the posterior densities are computed/estimated. a. A fixed regular grid used in the point-mass filter. b. Randomly allocated points following the regular PF equations. c. Points randomly allocated to vertical parallel lines (which themselves are randomly located) used in the DPF.

compared to the DRPA-PF, the DPF allows a simpler scheme for particle routing and actually treats each processing element as a particle in the particle routing. Second, the DPF does not have the efficiency decrease problem of the DRPA-PF. Given a PF with parallel structure, it works most efficiently if each processing element handles the same number of particles. However, the efficiency of the DRPA-PF usually decreases, since the numbers of particles produced by each processing element are not evenly but randomly distributed among the processing elements. Third, it will be verified by two numerical examples that, the DPF has the potential to achieve in a shorter execution time the same level of performance as the bootstrap PF. In contrast, the DRNA-PF actually trades the PF performance for the speed improvement [5]. Besides, the level of parallelism of the DPF can be further increased in two ways so that the execution time of the parallel implementation of the DPF can be further shortened; the first one is to utilize any of the distributed resampling algorithms proposed in [5, 22] to perform the first resampling of the DPF, and the other is based on an extension of the DPF. As a result, the DPF is a new option for the application of PFs in real-time systems and the parallel implementation of PFs.

The rest of the paper is organized as follows. The problem formulation is given in Section II. In Section III, the DPF algorithm is first derived and then summarized, and finally some issues regarding the implementation of the DPF are discussed. In Section IV, some discussions about the DPF and its comparison with the existing distributed PFs are made. In Section V, two numerical examples are elaborated to show the efficacy of the DPF. Finally, we conclude the paper in Section VI.

\section{Problem Formulation}

\section{A. Intuitive preview}

The formulas for particle filtering tend to look complex, and it may be easy to get lost in indices and update expressions. Let us therefore provide a simple and intuitive preview to get the main ideas across.

Filtering is about determining the posterior densities of the states. If the state is two-dimensional with components $x$ and $z$, say, the density is a surface over the $x-z$ plane, see Fig. 1. One way to estimate the density is to fix $M$ points in the plane in a regular grid (Fig. 1.a) and update the values of the density according to Bayesian formulas. This is known as the point-mass filter $[3,6]$. Another way is to throw $M$ points at the plane at random (Fig 1.b), and let them move to important places in the plane, and update the values of the densities at the chosen points, using Bayesian formulas. This is a simplified view of what happens in the regular PF. A third way is illustrated in Fig 1.c: Let the points move to well chosen locations, but restrict them to be aligned parallel to one of the axes (the $z$-axis in the plot). The parallel lines can move freely, as can the points on the lines, but there is a restriction of the pattern as depicted. The algorithm we develop in this paper (DPF) gives both the movements of the lines and the positions of the points on the lines, and the density values at the chosen points, by application of Bayesian formulas.

It is well known that the regular PF outperforms the pointmass filter with the same number of points, since it can concentrate them to important areas. One would thus expect that the DPF would give worse accuracy than the regular PF with the same number of points, since it is less "flexible" in the allocation of points. On the other hand, the structure might allow more efficient ways of calculating new point locations and weights. That is what we will develop and study in the following sections.

\section{B. Problem statement}

Consider system (1). Suppose that the state $\xi_{t}$ can be decomposed as

$$
\xi_{t}=\left[\begin{array}{l}
x_{t} \\
z_{t}
\end{array}\right]
$$

and accordingly that system (1) can be decomposed as

$$
\begin{aligned}
x_{t+1} & =f_{t}^{x}\left(x_{t}, z_{t}, v_{t}^{x}\right) \\
z_{t+1} & =f_{t}^{z}\left(x_{t}, z_{t}, v_{t}^{z}\right) \\
y_{t} & =h_{t}\left(x_{t}, z_{t}, e_{t}\right)
\end{aligned}
$$

where $x_{t} \in \mathbb{R}^{n_{x}}, z_{t} \in \mathbb{R}^{n_{z}}$, and $v_{t}=\left[\left(v_{t}^{x}\right)^{T}\left(v_{t}^{z}\right)^{T}\right]^{T}$ with $v_{t}^{x} \in \mathbb{R}^{n_{v^{x}}}$ and $v_{t}^{z} \in \mathbb{R}^{n_{v^{z}}}$. In the following, it is assumed for convenience that the probability densities $p\left(x_{0}\right), p\left(z_{0} \mid x_{0}\right)$ and for $t \geq 0, p\left(x_{t+1} \mid x_{t}, z_{t}\right), p\left(z_{t+1} \mid x_{t: t+1}, z_{t}\right)$ and $p\left(y_{t} \mid x_{t}, z_{t}\right)$ are known.

In this paper, we will study the filtering problem of recursively estimating the posterior density $p\left(z_{t}, x_{0: t} \mid y_{0: t}\right)$. According to the following factorization

$$
p\left(z_{t}, x_{0: t} \mid y_{0: t}\right)=p\left(z_{t} \mid x_{0: t}, y_{0: t}\right) p\left(x_{0: t} \mid y_{0: t}\right)
$$


where $x_{0: t} \triangleq\left\{x_{0}, \ldots, x_{t}\right\}$, the filtering problem (4) can be split into two nested sub-problems:

1) recursively estimating the density $p\left(x_{0: t} \mid y_{0: t}\right)$;

2) recursively estimating the density $p\left(z_{t} \mid x_{0: t}, y_{0: t}\right)$.

That the two sub-problems are nested can be seen from Fig. 2 where we have sketched the five steps used to derive the recurrence relation of the conceptual solution to the filtering problem (4). Since there is in general no analytic solution to the filtering problem (4), a numerical algorithm, i.e., the DPF is introduced to provide recursively the empirical approximations to $p\left(x_{0: t} \mid y_{0: t}\right)$ and $p\left(z_{t} \mid x_{0: t}, y_{0: t}\right)$. The DPF actually handles the two nested sub-problems using PFs. Roughly speaking, the DPF solves the first sub-problem using a PF with $N_{x}$ particles $\left(x_{0: t}^{(i)}, i=1, \ldots, N_{x}\right)$ to estimate $p\left(x_{0: t} \mid y_{0: t}\right)$. Then the DPF handles the second sub-problem using $N_{x}$ PFs with $N_{z}$ particles each to estimate $p\left(z_{t} \mid x_{0: t}^{(i)}, y_{0: t}\right), i=1, \ldots, N_{x}$. As a result of the nestedness of the two sub-problems, it will be seen later that the steps of the PF used to estimate $p\left(x_{0: t} \mid y_{0: t}\right)$ is nested with that of the $N_{x}$ PFs used to estimate $p\left(z_{t} \mid x_{0: t}^{(i)}, y_{0: t}\right)$.

Remark 2.1: The idea of decomposing the state into two parts and accordingly splitting the filtering problem into two nested sub-problems is not new. Actually, it has been used in the Rao-Blackwellized PF (RBPF); see, e.g, $[1,8,9,11,13$, 25]. However, the RBPF imposes certain tractable substructure assumption on the system considered and hence solves one of the sub-problem with a number of optimal filters, such as the Kalman filter [19] or the HMM filter [24]. In particular, the filtering problem (4) has been previously studied in [25] where system (3) is assumed to be conditionally (on $x_{t}$ ) linear in $z_{t}$ and subject to Gaussian noise. Due to these assumptions, the state $z_{t}$ of system (3) is marginalized out by using the Kalman filter. However, since there is no tractable substructure assumption made on system (3) in this paper, no part of the state $\xi_{t}$ is analytically tractable as was the case in $[1,8,9,11$, $13,25]$.

In the following, let $\tilde{x} \sim p(x)$ denote that $\tilde{x}$ is a sample drawn from the density $p(x)$ of the random variable $x$, let $\mathcal{N}(m, \Sigma)$ denote the (multivariate) Gaussian probability density with mean vector $m$ and covariance matrix $\Sigma$ and let $\mathrm{P}(A)$ denote the probability of the event $A$. For convenience, for each $i=1, \ldots, N, \alpha_{i}=\beta_{i} / \sum_{j=1}^{N} \beta_{j}$ is denoted by

$$
\alpha_{i} \propto \beta_{i} ; \quad \sum_{i=1}^{N} \alpha_{i}=1
$$

where $N$ is a natural number, $\alpha_{i}, \beta_{i}, i=1, \ldots, N$, are positive real numbers, and $\alpha_{i} \propto \beta_{i}$ denotes that $\alpha_{i}$ is proportional to $\beta_{i}$.

\section{DeCEntralized PARTICLE FILTER}

In this section, the DPF algorithm is first derived and then summarized. Finally, some issues regarding the implementation of the DPF are discussed.

We here make a comment regarding some notations used

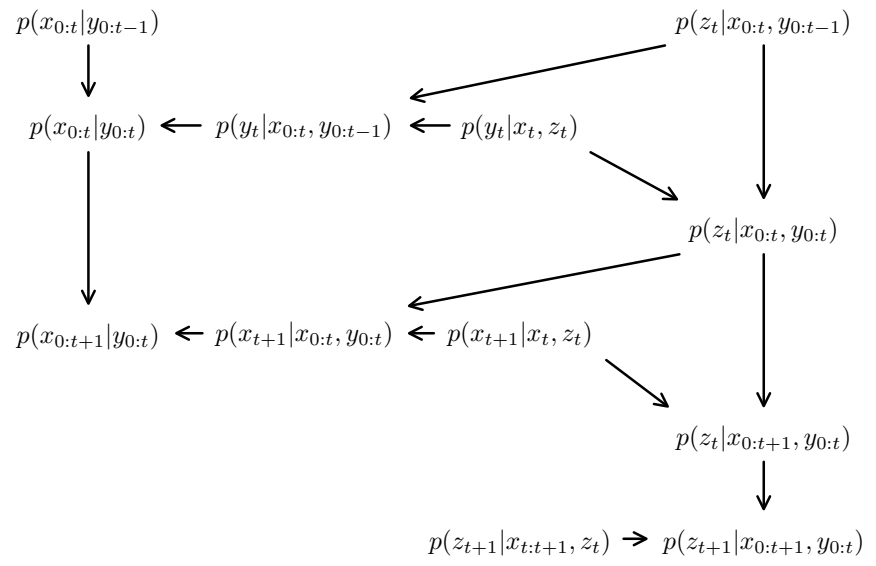

Fig. 2. Sketch of the steps used to derive the conceptual solution to the filtering problem (4). Assume that the probability densities $p\left(x_{0}\right), p\left(z_{0} \mid x_{0}\right)$ and for $t \geq 0, p\left(x_{t+1} \mid x_{t}, z_{t}\right), p\left(z_{t+1} \mid x_{t: t+1}, z_{t}\right)$ and $p\left(y_{t} \mid x_{t}, z_{t}\right)$ are known. With a slight abuse of notation let $p\left(x_{0} \mid y_{0:-1}\right)=p\left(x_{0}\right)$ and $p\left(z_{0} \mid x_{0}, y_{0:-1}\right)=p\left(z_{0} \mid x_{0}\right)$. Then five steps are needed to derive the recurrence relation of the conceptual solution to the filtering problem (4). The specific relations between the different densities can be obtained by straightforward application of Bayesian formulas and thus are omitted. Instead, the notation $p_{1}(\cdot) \rightarrow p_{2}(\cdot)$ is used to indicate that the calculation of the density $p_{2}(\cdot)$ makes use of the knowledge of the density $p_{1}(\cdot)$.

throughout the paper. Suppose we have

$$
P_{N}\left(d \xi_{t}\right)=\frac{1}{N} \sum_{i=1}^{N} \delta_{\xi_{t}^{(i)}}\left(d \xi_{t}\right)
$$

where $\delta_{\xi_{t}^{(i)}}(A)$ is a Dirac measure for a given $\xi_{t}^{(i)}$ and a measurable set $A$. Then the expectation of any test function $g\left(\xi_{t}\right)$ with respect to $p\left(\xi_{t}\right)$ can be approximated by

$$
\int g\left(\xi_{t}\right) p\left(\xi_{t}\right) d \xi_{t} \approx \int g\left(\xi_{t}\right) P_{N}\left(d \xi_{t}\right)=\frac{1}{N} \sum_{i=1}^{N} g\left(\xi_{t}^{(i)}\right)
$$

Although the distribution $P_{N}\left(d \xi_{t}\right)$ does not have a well defined density with respect to the Lebesgue measure, it is a common convention in the particle filtering community [2, 15] to use

$$
p_{N}\left(\xi_{t}\right)=\frac{1}{N} \sum_{i=1}^{N} \delta\left(\xi_{t}-\xi_{t}^{(i)}\right)
$$

where $\delta(\cdot)$ is a Dirac delta function, as if it is an empirical approximation of the probability density $p\left(\xi_{t}\right)$ with respect to the Lebesgue measure. The notations like (8) and the corresponding terms mentioned above are, in the most rigorous mathematical sense, not correct. However, they enable one to avoid the use of measure theory which indeed simplifies the representation a lot especially when a theoretical convergence proof is not the concern.

\section{A. Derivation of the DPF algorithm}

First, we initialize the particles $\tilde{x}_{0}^{(i)} \sim p\left(x_{0}\right), i=1, \ldots, N_{x}$, and for each $\tilde{x}_{0}^{(i)}$, the particles $\tilde{z}_{0}^{(i, j)} \sim p\left(z_{0} \mid \tilde{x}_{0}^{(i)}\right), j=$ $1, \ldots, N_{z}$. Then the derivation of the DPF algorithm will be completed in two steps by the induction principle. In the first 
step, we show that Inductive Assumptions 3.1 to 3.3 to be introduced below hold at $t=1$. In the second step, assume that Inductive Assumptions 3.1 to 3.3 hold at $t$ for $t \geq 1$, then we show that Inductive Assumptions 3.1 to 3.3 hold recursively at $t+1$

In the following, we first introduce Inductive Assumptions 3.1 to 3.3 at $t$.

Assumption 3.1: The DPF produce $N_{x}$ particles $x_{0: t-1}^{(i)}, i=$ $1, \ldots, N_{x}$ and an unweighted empirical approximation of $p\left(x_{0: t-1} \mid y_{0: t-1}\right)$ as

$$
\tilde{p}_{N_{x}}\left(x_{0: t-1} \mid y_{0: t-1}\right)=\frac{1}{N_{x}} \sum_{i=1}^{N_{x}} \delta\left(x_{0: t-1}-x_{0: t-1}^{(i)}\right)
$$

and for each path $x_{0: t-1}^{(i)}, i=1, \ldots, N_{x}$, the DPF produce $N_{z}$ particles $\bar{z}_{t-1}^{(i, j)}, j=1, \ldots, N_{z}$, and a weighted empirical approximation of $p\left(z_{t-1} \mid x_{0: t-1}^{(i)}, y_{0: t-1}\right)$ as

$$
p_{N_{z}}\left(z_{t-1} \mid x_{0: t-1}^{(i)}, y_{0: t-1}\right)=\sum_{j=1}^{N_{z}} \bar{q}_{t-1}^{(i, j)} \delta\left(z_{t-1}-\bar{z}_{t-1}^{(i, j)}\right)
$$

where $\bar{q}_{t-1}^{(i, j)}$ is the importance weight and its definition will be given in the derivation.

Assumption 3.2: The particles $\tilde{x}_{t}^{(i)}, i=1, \ldots, N_{x}$, are generated according to the proposal function $\pi\left(x_{t} \mid x_{0: t-1}^{(i)}, y_{0: t-1}\right)$ and for each $\tilde{x}_{t}^{(i)}, i=1, \ldots, N_{x}$, the particles $\tilde{z}_{t}^{(i, j)}, j=$ $1, \ldots, N_{z}$, are generated according to the proposal function $\pi\left(z_{t} \mid \tilde{x}_{0: t}^{(i)}, y_{0: t-1}\right)$ where $\tilde{x}_{0: t}^{(i)} \triangleq\left(x_{0: t-1}^{(i)}, \tilde{x}_{t}^{(i)}\right)$.

Assumption 3.3: For each $i=1, \ldots, N_{x}$, an approximation $p_{N_{z}}\left(y_{t} \mid \tilde{x}_{0: t}^{(i)}, y_{0: t-1}\right)$ of $p\left(y_{t} \mid \tilde{x}_{0: t}^{(i)}, y_{0: t-1}\right)$ can be obtained as

$$
\begin{aligned}
p_{N_{z}}\left(y_{t} \mid \tilde{x}_{0: t}^{(i)}, y_{0: t-1}\right) & \\
= & \sum_{j=1}^{N_{z}} \tilde{r}_{t}^{(i, j)} p\left(y_{t} \mid \tilde{x}_{t}^{(i)}, \tilde{z}_{t}^{(i, j)}\right) / \sum_{l=1}^{N_{z}} \tilde{r}_{t}^{(i, l)}
\end{aligned}
$$

with $\tilde{r}_{t}^{(i, j)}=\tilde{p}_{N_{z}}\left(\tilde{z}_{t}^{(i, j)} \mid \tilde{x}_{0: t}^{(i)}, y_{0: t-1}\right) / \pi\left(\tilde{z}_{t}^{(i, j)} \mid \tilde{x}_{0: t}^{(i)}, y_{0: t-1}\right)$, where $\tilde{p}_{N_{z}}\left(\tilde{z}_{t}^{(i, j)} \mid \tilde{x}_{0: t}^{(i)}, y_{0: t-1}\right)$ is an approximation of $p\left(\tilde{z}_{t}^{(i, j)} \mid \tilde{x}_{0: t}^{(i)}, y_{0: t-1}\right)$ and its definition will be given in the derivation.

Remark 3.1: In Inductive Assumptions 3.1 and 3.3, $\tilde{p}_{N_{x}}\left(x_{0: t-1} \mid y_{0: t-1}\right)$ and $p_{N_{z}}\left(z_{t-1} \mid x_{0: t-1}^{(i)}, y_{0: t-1}\right)$ are empirical approximations of $p\left(x_{0: t-1} \mid y_{0: t-1}\right)$ and $p\left(z_{t-1} \mid x_{0: t-1}^{(i)}, y_{0: t-1}\right)$, respectively. These approximations should of course be as close to the true underlying density as possible. This closeness is typically assessed via some test function $g_{t-1}(\cdot)$ used in the following sense,

$$
\begin{aligned}
I\left(g_{t-1}\right)= & \int g_{t-1}\left(z_{t-1}, x_{0: t-1}\right) \\
& \times p\left(z_{t-1}, x_{0: t-1} \mid y_{0: t-1}\right) d z_{t-1} d x_{0: t-1}
\end{aligned}
$$

where $g_{t-1}: \mathbb{R}^{n_{z}} \times \mathbb{R}^{t \times n_{x}} \rightarrow \mathbb{R}$ is a test function. With the empirical approximations defined in (9) and (10), an estimate $I_{N_{x}, N_{z}}\left(g_{t-1}\right)$ of $I\left(g_{t-1}\right)$ is obtained as follows

$$
I_{N_{x}, N_{z}}\left(g_{t-1}\right)=\frac{1}{N_{x}} \sum_{i=1}^{N_{x}} \sum_{j=1}^{N_{z}} \bar{q}_{t-1}^{(i, j)} g_{t-1}\left(\bar{z}_{t-1}^{(i, j)}, x_{0: t-1}^{(i)}\right)
$$

A convergence result formalizing the "closeness" of (13) to (12), as $N_{x}$ and $N_{z}$ tend to infinity, will be in line with our earlier convergence results [18] of the PF for rather arbitrary unbounded test functions.

Then in the first step we should show that Inductive Assumptions 3.1 to 3.3 hold at $t=1$. However, since the first step is exactly the same as the second step, we only consider the second step here due to the space limitation. In the second step, assume that Inductive Assumptions 3.1 to 3.3 hold at $t$, then we will show in seven steps that Inductive Assumptions 3.1 to 3.3 recursively hold at $t+1$.

1) Measurement update of $x_{0: t}$ based on $y_{t}$

By using importance sampling, this step aims to derive a weighted empirical approximation of $p\left(x_{0: t} \mid y_{0: t}\right)$. Note that an unweighted empirical approximation $\tilde{p}_{N_{x}}\left(x_{0: t-1} \mid y_{0: t-1}\right)$ of $p\left(x_{0: t-1} \mid y_{0: t-1}\right)$ has been given as (9) in Inductive Assumption 3.1, then simple calculation shows that the following equation holds approximately

$$
\begin{aligned}
p\left(x_{0: t} \mid y_{0: t}\right) \propto \tilde{p}_{N_{x}}\left(x_{0: t-1} \mid y_{0: t-1}\right) p\left(x_{t} \mid x_{0: t-1}, y_{0: t-1}\right) & \\
& \times p\left(y_{t} \mid x_{0: t}, y_{0: t-1}\right)
\end{aligned}
$$

From Inductive Assumptions 3.1 and 3.2, $x_{0: t-1}^{(i)}, i=$ $1, \ldots, N_{x}$, can be regarded as samples drawn from $\tilde{p}_{N_{x}}\left(x_{0: t-1} \mid y_{0: t-1}\right)$, and for $i=1, \ldots, N_{x}, \tilde{x}_{t}^{(i)}$ is the sample drawn from $\pi\left(x_{t} \mid x_{0: t-1}^{(i)}, y_{0: t-1}\right)$. Therefore $\tilde{p}_{N_{x}}\left(x_{0: t-1} \mid y_{0: t-1}\right) \pi\left(x_{t} \mid x_{0: t-1}^{(i)}, y_{0: t-1}\right)$ can be treated as the importance function. On the other hand, from (10) and

$$
\begin{aligned}
& p\left(x_{t} \mid x_{0: t-1}, y_{0: t-1}\right) \\
& =\int p\left(z_{t-1} \mid x_{0: t-1}, y_{0: t-1}\right) p\left(x_{t} \mid x_{t-1}, z_{t-1}\right) d z_{t}
\end{aligned}
$$

an approximation $\quad p_{N_{z}}\left(\tilde{x}_{t}^{(i)} \mid x_{0: t-1}^{(i)}, y_{0: t-1}\right) \quad$ of $p\left(\tilde{x}_{t}^{(i)} \mid x_{0: t-1}^{(i)}, y_{0: t-1}\right)$ can be obtained as

$$
\begin{aligned}
p_{N_{z}}\left(\tilde{x}_{t}^{(i)} \mid x_{0: t-1}^{(i)}, y_{0: t-1}\right) & \\
= & \sum_{j=1}^{N_{z}} \bar{q}_{t-1}^{(i, j)} p\left(\tilde{x}_{t}^{(i)} \mid x_{t-1}^{(i)}, \bar{z}_{t-1}^{(i, j)}\right)
\end{aligned}
$$

Moreover, an approximation $p_{N_{z}}\left(y_{t} \mid \tilde{x}_{0: t}^{(i)}, y_{0: t-1}\right)$ of $p\left(y_{t} \mid \tilde{x}_{0: t}^{(i)}, y_{0: t-1}\right)$ has been given as (11) in Inductive Assumption 3.3.

Now using the importance sampling yields a weighted empirical approximation of $p\left(x_{0: t} \mid y_{0: t}\right)$ as

$$
p_{N_{x}}\left(x_{0: t} \mid y_{0: t}\right)=\sum_{i=1}^{N_{x}} w_{t}^{(i)} \delta\left(x_{0: t}-\tilde{x}_{0: t}^{(i)}\right)
$$

where $w_{t}^{(i)}$ is evaluated according to

$$
\begin{gathered}
w_{t}^{(i)} \propto \frac{p_{N_{z}}\left(y_{t} \mid \tilde{x}_{0: t}^{(i)}, y_{0: t-1}\right) p_{N_{z}}\left(\tilde{x}_{t}^{(i)} \mid x_{0: t-1}^{(i)}, y_{0: t-1}\right)}{\pi\left(\tilde{x}_{t}^{(i)} \mid x_{0: t-1}^{(i)}, y_{0: t-1}\right)} \\
\sum_{i=1}^{N_{x}} w_{t}^{(i)}=1
\end{gathered}
$$


2) Resampling of $\left\{\tilde{x}_{0: t}^{(i)}, \tilde{z}_{t}^{(i, 1)}, \tilde{r}_{t}^{(i, 1)}, \ldots, \tilde{z}_{t}^{\left(i, N_{z}\right)}, \tilde{r}_{t}^{\left(i, N_{z}\right)}\right\}, i=$ $1, \ldots, N_{x}$

By using resampling, this step aims to derive an unweighted empirical approximation of $p\left(x_{0: t} \mid y_{0: t}\right)$.

Resample $N_{x}$ times from the discrete distribution over $\left\{\left\{\tilde{x}_{0: t}^{(i)}, \tilde{z}_{t}^{(i, 1)}, \tilde{r}_{t}^{(i, 1)}, \ldots, \tilde{z}_{t}^{\left(i, N_{z}\right)}, \tilde{r}_{t}^{\left(i, N_{z}\right)}\right\}, i=1, \ldots, N_{x}\right\}$ with probability mass $w_{t}^{(i)}$ associated with the element $\left\{\tilde{x}_{0: t}^{(i)}, \tilde{z}_{t}^{(i, 1)}, \tilde{r}_{t}^{(i, 1)}, \ldots, \tilde{z}_{t}^{\left(i, N_{z}\right)}, \tilde{r}_{t}^{\left(i, N_{z}\right)}\right\}$ to generate samples $\left\{x_{0: t}^{(i)}, \bar{z}_{t}^{(i, 1)}, r_{t}^{(i, 1)}, \ldots, \bar{z}_{t}^{\left(i, N_{z}\right)}, r_{t}^{\left(i, N_{z}\right)}\right\}, i=1, \ldots, N_{x}$, so that for any $m$,

$$
\begin{aligned}
& \mathrm{P}\left\{\left\{x_{0: t}^{(m)}, \bar{z}_{t}^{(m, 1)}, r_{t}^{(m, 1)}, \ldots, \bar{z}_{t}^{\left(m, N_{z}\right)}, r_{t}^{\left(m, N_{z}\right)}\right\}=\right. \\
& \left.\left\{\tilde{x}_{0: t}^{(i)}, \tilde{z}_{t}^{(i, 1)}, \tilde{r}_{t}^{(i, 1)}, \ldots, \tilde{z}_{t}^{\left(i, N_{z}\right)}, \tilde{r}_{t}^{\left(i, N_{z}\right)}\right\}\right\}=w_{t}^{(i)}
\end{aligned}
$$

where $r_{t}^{(i, j)}$ can be defined as $r_{t}^{(i, j)}=$ $\tilde{p}_{N_{z}}\left(\bar{z}_{t}^{(i, j)} \mid x_{0: t}^{(i)}, y_{0: t-1}\right) / \pi\left(\bar{z}_{t}^{(i, j)} \mid x_{0: t}^{(i)}, y_{0: t-1}\right) \quad$ according to the definition of $\tilde{r}_{t}^{(i, j)}$ in Inductive Assumption 3.3 (See Section III-C1 for more detailed explanation).

As a result, it follows from (17) that an unweighted empirical approximation of $p\left(x_{0: t} \mid y_{0: t}\right)$ is obtained as

$$
\tilde{p}_{N_{x}}\left(x_{0: t} \mid y_{0: t}\right)=\frac{1}{N_{x}} \sum_{i=1}^{N_{x}} \delta\left(x_{0: t}-x_{0: t}^{(i)}\right)
$$

3) Measurement update of $z_{t}$ based on $y_{t}$

By using importance sampling, this step aims to derive a weighted empirical approximation of $p\left(z_{t} \mid x_{0: t}^{(i)}, y_{0: t}\right)$. Simple calculation shows that

$$
p\left(z_{t} \mid x_{0: t}^{(i)}, y_{0: t}\right) \propto p\left(z_{t} \mid x_{0: t}^{(i)}, y_{0: t-1}\right) p\left(y_{t} \mid x_{t}^{(i)}, z_{t}\right)
$$

From Inductive Assumption 3.2, for each $x_{t}^{(i)}, i=$ $1, \ldots, N_{x}$, the particles $\bar{z}_{t}^{(i, j)}, j=1, \ldots, N_{z}$, are generated from the proposal function $\pi\left(z_{t} \mid x_{0: t}^{(i)}, y_{0: t-1}\right)$. Therefore $\pi\left(z_{t} \mid x_{0: t}^{(i)}, y_{0: t-1}\right)$ is chosen as the importance function. On the other hand, note that an approximation $\tilde{p}_{N_{z}}\left(\bar{z}_{t}^{(i, j)} \mid x_{0: t}^{(i)}, y_{0: t-1}\right)$ of $p\left(\bar{z}_{t}^{(i, j)} \mid x_{0: t}^{(i)}, y_{0: t-1}\right)$ has been given in Inductive Assumption 3.3.

Then using importance sampling and also noting the definition of $r_{t}^{(i, j)}$ yields, for each $i=1, \ldots, N_{x}$, a weighted empirical approximation of $p\left(z_{t} \mid x_{0: t}^{(i)}, y_{0: t}\right)$ as

$$
p_{N_{z}}\left(z_{t} \mid x_{0: t}^{(i)}, y_{0: t}\right)=\sum_{j=1}^{N_{z}} \bar{q}_{t}^{(i, j)} \delta\left(z_{t}-\bar{z}_{t}^{(i, j)}\right)
$$

where $\bar{q}_{t}^{(i, j)}$ is evaluated according to

$$
\bar{q}_{t}^{(i, j)} \propto p\left(y_{t} \mid x_{t}^{(i)}, \bar{z}_{t}^{(i, j)}\right) r_{t}^{(i, j)} ; \quad \sum_{j=1}^{N_{z}} \bar{q}_{t}^{(i, j)}=1
$$

4) Generation of particles $\tilde{x}_{t+1}^{(i)}, i=1, \ldots, N_{x}$

Assume that the particles $\tilde{x}_{t+1}^{(i)}, i=1, \ldots, N_{x}$, are generated according to the proposal function $\pi\left(x_{t+1} \mid x_{0: t}^{(i)}, y_{0: t}\right)$.

5) Measurement update of $z_{t}$ based on $x_{t+1}$

By using importance sampling, this step aims to derive a weighted empirical approximation of $p\left(z_{t} \mid \tilde{x}_{0: t+1}^{(i)}, y_{0: t}\right)$.
Simple calculation shows that

$$
\begin{gathered}
p\left(z_{t} \mid \tilde{x}_{0: t+1}^{(i)}, y_{0: t}\right) \propto p\left(z_{t} \mid x_{0: t}^{(i)}, y_{0: t-1}\right) p\left(y_{t} \mid x_{t}^{(i)}, z_{t}\right) \\
\times p\left(\tilde{x}_{t+1}^{(i)} \mid x_{t}^{(i)}, z_{t}\right)
\end{gathered}
$$

Analogously to step 3$)$, choose $\pi\left(z_{t} \mid x_{0: t}^{(i)}, y_{0: t-1}\right)$ as the importance function and note that $\tilde{p}_{N_{z}}\left(\bar{z}_{t}^{(i, j)} \mid x_{0: t}^{(i)}, y_{0: t-1}\right)$ is an approximation of $p\left(\bar{z}_{t}^{(i, j)} \mid x_{0: t}^{(i)}, y_{0: t-1}\right)$. Using importance sampling and also noting the definition of $r_{t}^{(i, j)}$ yields, for each $i=1, \ldots, N_{x}$, a weighted empirical approximation of $p\left(z_{t} \mid \tilde{x}_{0: t+1}^{(i)}, y_{0: t}\right)$ as

$$
p_{N_{z}}\left(z_{t} \mid \tilde{x}_{0: t+1}^{(i)}, y_{0: t}\right)=\sum_{j=1}^{N_{z}} q_{t}^{(i, j)} \delta\left(z_{t}-\bar{z}_{t}^{(i, j)}\right)
$$

where $\tilde{x}_{0: t+1}^{(i)} \triangleq\left(x_{0: t}^{(i)}, \tilde{x}_{t+1}^{(i)}\right)$ and $q_{t}^{(i, j)}$ is evaluated according to

$$
\begin{gathered}
q_{t}^{(i, j)} \propto p\left(y_{t} \mid x_{t}^{(i)}, \bar{z}_{t}^{(i, j)}\right) p\left(\tilde{x}_{t+1}^{(i)} \mid x_{t}^{(i)}, \bar{z}_{t}^{(i, j)}\right) r_{t}^{(i, j)} ; \\
\sum_{j=1}^{N_{z}} q_{t}^{(i, j)}=1
\end{gathered}
$$

6) Resampling of the particles $\bar{z}_{t}^{(i, j)}, i=1, \ldots, N_{x}, j=$ $1, \ldots, N_{z}$

By using resampling, this step aims to derive an unweighted empirical approximation of $p\left(z_{t} \mid \tilde{x}_{0: t+1}^{(i)}, y_{0: t}\right)$.

For each $i=1, \ldots, N_{x}$, resample $N_{z}$ times from the discrete distribution over $\left\{\bar{z}_{t}^{(i, j)}, j=1, \ldots, N_{z}\right\}$ with probability mass $q_{t}^{(i, j)}$ associated with the element $\bar{z}_{t}^{(i, j)}$ to generate samples $\left\{z_{t}^{(i, j)}, j=1, \ldots, N_{z}\right\}$, so that for any $m$,

$$
\mathrm{P}\left\{z_{t}^{(i, m)}=\bar{z}_{t}^{(i, j)}\right\}=q_{t}^{(i, j)}
$$

As a result, it follows from (25) that for each $i=$ $1, \ldots, N_{x}$, an unweighted empirical approximation of $p\left(z_{t} \mid \tilde{x}_{0: t+1}^{(i)}, y_{0: t}\right)$ is as follows

$$
\tilde{p}_{N_{z}}\left(z_{t} \mid \tilde{x}_{0: t+1}^{(i)}, y_{0: t}\right)=\frac{1}{N_{z}} \sum_{j=1}^{N_{z}} \delta\left(z_{t}-z_{t}^{(i, j)}\right)
$$

7) Generation of particles $\tilde{z}_{t+1}^{(i, j)}, i=1, \ldots, N_{x}, j=1, \ldots, N_{z}$ Assume that for each $\tilde{x}_{t+1}^{(i)}, i=1, \ldots, N_{x}$, the particles $\tilde{z}_{t+1}^{(i, j)}, j=1, \ldots, N_{z}$, are generated according to the proposal function $\pi\left(z_{t+1} \mid \tilde{x}_{0: t+1}^{(i)}, y_{0: t}\right)$.

By using importance sampling, we try to derive a weighted empirical approximation of $p\left(z_{t+1} \mid \tilde{x}_{0: t+1}^{(i)}, y_{0: t}\right)$. First, choose $\pi\left(z_{t+1} \mid \tilde{x}_{0: t+1}^{(i)}, y_{0: t}\right)$ as the importance function. Then from (28) and

$$
\begin{aligned}
& p\left(z_{t+1} \mid x_{0: t+1}, y_{0: t}\right) \\
& \quad=\int p\left(z_{t} \mid x_{0: t+1}, y_{0: t}\right) p\left(z_{t+1} \mid x_{t: t+1}, z_{t}\right) d z_{t}
\end{aligned}
$$

an approximation of $p\left(z_{t+1} \mid \tilde{x}_{0: t+1}^{(i)}, y_{0: t}\right), i=1, \ldots, N_{x}$, can be obtained as

$$
\begin{aligned}
& \tilde{p}_{N_{z}}\left(z_{t+1} \mid \tilde{x}_{0: t+1}^{(i)}, y_{0: t}\right) \\
& \quad=\frac{1}{N_{z}} \sum_{l=1}^{N_{z}} p\left(z_{t+1} \mid \tilde{x}_{t: t+1}^{(i)}, z_{t}^{(i, l)}\right)
\end{aligned}
$$


Now using importance sampling yields that for $i=$ $1, \ldots, N_{x}$, a weighted empirical approximation of $p\left(z_{t+1} \mid \tilde{x}_{0: t+1}^{(i)}, y_{0: t}\right)$ as follows

$$
\begin{aligned}
& p_{N_{z}}\left(z_{t+1} \mid \tilde{x}_{0: t+1}^{(i)}, y_{0: t}\right) \\
& =\sum_{j=1}^{N_{z}} \tilde{r}_{t+1}^{(i, j)} \delta\left(z_{t+1}-\tilde{z}_{t+1}^{(i, j)}\right) / \sum_{l=1}^{N_{z}} \tilde{r}_{t+1}^{(i, l)}
\end{aligned}
$$

where $\tilde{r}_{t+1}^{(i, j)}$ is evaluated according to

$$
\tilde{r}_{t+1}^{(i, j)}=\tilde{p}_{N_{z}}\left(\tilde{z}_{t+1}^{(i, j)} \mid \tilde{x}_{0: t+1}^{(i)}, y_{0: t}\right) / \pi\left(\tilde{z}_{t+1}^{(i, j)} \mid \tilde{x}_{0: t+1}^{(i)}, y_{0: t}\right)
$$

Finally, from (31) and

$$
\begin{aligned}
& p\left(y_{t+1} \mid x_{0: t+1}, y_{0: t}\right) \\
& \quad=\int p\left(z_{t+1} \mid x_{0: t+1}, y_{0: t}\right) p\left(y_{t+1} \mid x_{t+1}, z_{t+1}\right) d z_{t+1}
\end{aligned}
$$

an approximation

$$
p_{N_{z}}\left(y_{t+1} \mid \tilde{x}_{0: t+1}^{(i)}, y_{0: t}\right)
$$

$p\left(y_{t+1} \mid \tilde{x}_{0: t+1}^{(i)}, y_{0: t}\right)$ can be obtained as (11) with $t$ replaced by $t+1$. In turn, it can be seen from (20), (22), step 4), step 7), and (32) that Inductive Assumptions 3.1 to 3.3 hold with $t$ replaced by $t+1$. Hence, we have completed the derivation of the DPF by the induction principle.

\section{B. Summary of the filtering algorithm}

\section{Initialization}

Initialize the particles $\tilde{x}_{0}^{(i)} \sim p\left(x_{0}\right), i=1, \ldots, N_{x}$, and for each $\tilde{x}_{0}^{(i)}$, the particles $\tilde{z}_{0}^{(i, j)} \sim p\left(z_{0} \mid \tilde{x}_{0}^{(i)}\right), j=1, \ldots, N_{z}$. With a slight abuse of notation let $p\left(x_{0}\right)=p_{N_{z}}\left(x_{0} \mid x_{0:-1}, y_{0:-1}\right)=$ $\pi\left(x_{0} \mid x_{0:-1}, y_{0:-1}\right)$ and $p\left(z_{0} \mid x_{0}\right)=\tilde{p}_{N_{z}}\left(z_{0} \mid x_{0}, y_{0:-1}\right)=$ $\pi\left(z_{0} \mid x_{0}, y_{0:-1}\right)$.

At each time instant $(t \geq 0)$

1) Measurement update of $x_{0: t}$ based on $y_{t}$

The importance weights $w_{t}^{(i)}, i=1, \ldots, N_{x}$, are evaluated according to (18).

2) Resampling of $\left\{\tilde{x}_{0: t}^{(i)}, \tilde{z}_{t}^{(i, 1)}, \tilde{r}_{t}^{(i, 1)} \ldots, \tilde{z}_{t}^{\left(i, N_{z}\right)}, \tilde{r}_{t}^{\left(i, N_{z}\right)}\right\}, i=$ $1, \ldots, N_{x}$

According to (19), resample $\left\{\tilde{x}_{0: t}^{(i)}, \tilde{z}_{t}^{(i, 1)}, \tilde{r}_{t}^{(i, 1)} \ldots, \tilde{z}_{t}^{\left(i, N_{z}\right)}, \tilde{r}_{t}^{\left(i, N_{z}\right)}\right\}, i=1, \ldots, N_{x}$, to generate samples $\left\{x_{0: t}^{(i)}, \bar{z}_{t}^{(i, 1)}, r_{t}^{(i, 1)} \ldots, \bar{z}_{t}^{\left(i, N_{z}\right)}, r_{t}^{\left(i, N_{z}\right)}\right\}$, $i=1, \ldots, N_{x}$, where for $t \geq 0, \tilde{r}_{t}^{(i, j)}$ is defined according to $(32)$.

3) Measurement update of $z_{t}$ based on $y_{t}$

For $i=1, \ldots, N_{x}$, the importance weights $\bar{q}_{t}^{(i, j)}, j=$ $1, \ldots, N_{z}$, are evaluated according to (23).

4) Generation of particles $\tilde{x}_{t+1}^{(i)}, i=1, \ldots, N_{x}$

For each $i=1, \ldots, N_{x}, \tilde{x}_{t+1}^{(i)}$ is generated according to the proposal function $\pi\left(x_{t+1} \mid x_{0: t}^{(i)}, y_{0: t}\right)$.

5) Measurement update of $z_{t}$ based on $x_{t+1}$

For $i=1, \ldots, N_{x}$, the importance weights $q_{t}^{(i, j)}, j=$ $1, \ldots, N_{z}$, are evaluated according to (26).

6) Resampling of the particles $\bar{z}_{t}^{(i, j)}, i=1, \ldots, N_{x}, j=$ $1, \ldots, N_{z}$

According to (27), for each $i=1, \ldots, N_{x}$, resample the particles $\bar{z}_{t}^{(i, j)}, j=1, \ldots, N_{z}$, to generate samples $z_{t}^{(i, j)}, j=1, \ldots, N_{z}$.
7) Generation of particles $\tilde{z}_{t+1}^{(i, j)}, i=1, \ldots, N_{x}, j=1, \ldots, N_{z}$ For each $i=1, \ldots, N_{x}$, the particles $\tilde{z}_{t+1}^{(i, j)}, j=$ $1, \ldots, N_{z}$, are generated according to the proposal function $\pi\left(z_{t+1} \mid \tilde{x}_{0: t+1}^{(i)}, y_{0: t}\right)$ where $\tilde{x}_{0: t+1}^{(i)} \triangleq\left(x_{0: t}^{(i)}, \tilde{x}_{t+1}^{(i)}\right)$.

\section{Implementation issues}

1) Two resampling steps: Unlike most of PFs in the literature, the DPF has two resampling steps, i.e., step 2) and step 6). Furthermore, the second resampling bears a parallel structure. This is because the particles $\bar{z}_{t}^{(i, j)}, i=1, \ldots, N_{x}$, $j=1, \ldots, N_{z}$, can be divided into $N_{x}$ independent groups in terms of the index $i$. Therefore, the second resampling can be implemented in parallel.

In the implementation of the first resampling, it would be helpful to note the following points. For $i=1, \ldots, N_{x}, \quad\left\{\tilde{r}_{t}^{(i, 1)}, \ldots, \tilde{r}_{t}^{\left(i, N_{z}\right)}\right\}$ is associated with $\left\{\tilde{x}_{0: t}^{(i)}, \tilde{z}_{t}^{(i, 1)}, \ldots, \tilde{z}_{t}^{\left(i, N_{z}\right)}\right\}$, according to the definition $\tilde{r}_{t}^{(i, j)}=$ $\tilde{p}_{N_{z}}\left(\tilde{z}_{t}^{(i, j)} \mid \tilde{x}_{0: t}^{(i)}, y_{0: t-1}\right) / \pi\left(\tilde{z}_{t}^{(i, j)} \mid \tilde{x}_{0: t}^{(i)}, y_{0: t-1}\right)$. Therefore, after resampling of $\left\{\tilde{x}_{0: t}^{(i)}, \tilde{z}_{t}^{(i, 1)}, \ldots, \tilde{z}_{t}^{\left(i, N_{z}\right)}\right\}, i=1, \ldots, N_{x}$, $\left\{\tilde{r}_{t}^{(i, 1)}, \ldots, \tilde{r}_{t}^{\left(i, N_{z}\right)}\right\}, i=1, \ldots, N_{x}$, should accordingly be resampled to obtain $\left\{r_{t}^{(i, 1)}, \ldots, r_{t}^{\left(i, N_{z}\right)}\right\}, i=1, \ldots, N_{x}$. According to the definition of $\tilde{r}_{t}^{(i, j)}, r_{t}^{(i, j)}$ can be defined as $r_{t}^{(i, j)}=\tilde{p}_{N_{z}}\left(\bar{z}_{t}^{(i, j)} \mid x_{0: t}^{(i)}, y_{0: t-1}\right) / \pi\left(\bar{z}_{t}^{(i, j)} \mid x_{0: t}^{(i)}, y_{0: t-1}\right)$. As a result, $\left\{\tilde{x}_{0: t}^{(i)}, \tilde{z}_{t}^{(i, 1)}, \tilde{r}_{t}^{(i, 1)}, \ldots, \tilde{z}_{t}^{\left(i, N_{z}\right)}, \tilde{r}_{t}^{\left(i, N_{z}\right)}\right\}, \quad i=$ $1, \ldots, N_{x}$, is resampled in step 2). Moreover, since the particles $x_{0: t-1}^{(i)}, i=1, \ldots, N_{x}$, will not be used in the future, it is actually only necessary to resample $\left\{\tilde{x}_{t}^{(i)}, \tilde{z}_{t}^{(i, 1)}, \tilde{r}_{t}^{(i, 1)}, \ldots, \tilde{z}_{t}^{\left(i, N_{z}\right)}, r_{t}^{\left(i, N_{z}\right)}\right\}, i=1, \ldots, N_{x}$, to generate $\left\{x_{t}^{(i)}, \bar{z}_{t}^{(i, 1)}, r_{t}^{(i, 1)}, \ldots, \bar{z}_{t}^{\left(i, N_{z}\right)}, r_{t}^{\left(i, N_{z}\right)}\right\}, i=1, \ldots, N_{x}$.

2) Construction of the proposal functions: Like [15] where the "prior" is chosen as the proposal function, we try to choose $p\left(x_{t+1} \mid x_{0: t}^{(i)}, y_{0: t}\right)$ and $p\left(z_{t+1} \mid \tilde{x}_{0: t+1}^{(i)}, y_{0: t}\right)$ as the proposal functions $\pi\left(x_{t+1} \mid x_{0: t}^{(i)}, y_{0: t}\right)$ and $\pi\left(z_{t+1} \mid \tilde{x}_{0: t+1}^{(i)}, y_{0: t}\right)$, respectively. Unlike [15], however, $p\left(x_{t+1} \mid x_{0: t}^{(i)}, y_{0: t}\right)$ and $p\left(z_{t+1} \mid \tilde{x}_{0: t+1}^{(i)}, y_{0: t}\right)$ are usually unknown. Therefore, we need to construct approximations to $p\left(x_{t+1} \mid x_{0: t}^{(i)}, y_{0: t}\right)$ and $p\left(z_{t+1} \mid \tilde{x}_{0: t+1}^{(i)}, y_{0: t}\right)$ such that the particles $\tilde{x}_{t+1}^{(i)}$ and $\tilde{z}_{t+1}^{(i, j)}$, $j=1, \ldots, N_{z}$, can be sampled from the approximations, respectively.

A convenient way to construct those approximations is given as follows. From (22), an approximation of $p\left(x_{t+1} \mid x_{0: t}^{(i)}, y_{0: t}\right)$ can be obtained as

$$
p_{N_{z}}\left(x_{t+1} \mid x_{0: t}^{(i)}, y_{0: t}\right)=\sum_{j=1}^{N_{z}} \bar{q}_{t}^{(i, j)} p\left(x_{t+1} \mid x_{t}^{(i)}, \bar{z}_{t}^{(i, j)}\right)
$$

In turn, a further approximation of $p\left(x_{t+1} \mid x_{0: t}^{(i)}, y_{0: t}\right)$ can be obtained as $\mathcal{N}\left(\bar{x}_{t+1}^{(i)}, \Sigma_{t+1}^{(i)}\right)$ with $\bar{x}_{t+1}^{(i)}$ and $\Sigma_{t+1}^{(i)}$, respectively, the mean and the covariance of the discrete distribution over $\left\{\tilde{x}_{t+1}^{(i, j)}, j=1, \ldots, N_{z}\right\}$ with probability mass $\bar{q}_{t}^{(i, j)}$ associated with the element $\tilde{x}_{t+1}^{(i, j)} \sim p\left(x_{t+1} \mid x_{t}^{(i)}, \bar{z}_{t}^{(i, j)}\right)$. Therefore, for $i=1, \ldots, N_{x}$, the particle $\tilde{x}_{t+1}^{(i)}$ can be generated from

$$
\pi\left(x_{t+1} \mid x_{0: t}^{(i)}, y_{0: t}\right)=\mathcal{N}\left(\bar{x}_{t+1}^{(i)}, \Sigma_{t+1}^{(i)}\right)
$$

On the other hand, from (28) and (29), an approximation $\tilde{p}_{N_{z}}\left(z_{t+1} \mid \tilde{x}_{0: t+1}^{(i)}, y_{0: t}\right)$ of $p\left(z_{t+1} \mid \tilde{x}_{0: t+1}^{(i)}, y_{0: t}\right)$ has already been 
given in (30). Then, it follows from (30) and the assumption that $p\left(z_{t+1} \mid x_{t: t+1}, z_{t}\right)$ is known that, for each $i=1, \ldots, N_{x}$, the particle $\tilde{z}_{t+1}^{(i, j)}, j=1, \ldots, N_{z}$ can be generated from

$$
\pi\left(z_{t+1} \mid \tilde{x}_{0: t+1}^{(i)}, y_{0: t}\right)=\tilde{p}_{N_{z}}\left(z_{t+1} \mid \tilde{x}_{0: t+1}^{(i)}, y_{0: t}\right)
$$

Remark 3.2: From (25) and (29), another approximation of $p\left(z_{t+1} \mid \tilde{x}_{0: t+1}^{(i)}, y_{0: t}\right)$ can be obtained as

$$
\begin{aligned}
& p_{N_{z}}\left(z_{t+1} \mid \tilde{x}_{0: t+1}^{(i)}, y_{0: t}\right) \\
& =\sum_{j=1}^{N_{z}} q_{t}^{(i, j)} p\left(z_{t+1} \mid \tilde{x}_{0: t+1}^{(i)}, \bar{z}_{t}^{(i, j)}, y_{0: t}\right)
\end{aligned}
$$

This observation shows that the PF used to estimate $p\left(z_{t} \mid x_{0: t}^{(i)}, y_{0: t}\right)$ is closely related to the so-called marginal PF [21]. A marginal PF would sample the particles $\tilde{z}_{t+1}^{(i, j)}$, $j=1, \ldots, N_{z}$, from

$$
\begin{aligned}
& \pi\left(z_{t+1} \mid \tilde{x}_{0: t+1}^{(i)}, y_{0: t}\right) \\
& =\sum_{j=1}^{N_{z}} q_{t}^{(i, j)} \pi\left(z_{t+1} \mid \tilde{x}_{0: t+1}^{(i)}, \bar{z}_{t}^{(i, j)}, y_{0: t}\right)
\end{aligned}
$$

According to [21], sampling from (38) is precisely equivalent to first resample the particles $\bar{z}_{t}^{(i, j)}, i=1, \ldots, N_{x}$, $j=1, \ldots, N_{z}$ according to (27) and then sample the particle $\tilde{z}_{t+1}^{(i, j)}$ from $\pi\left(z_{t+1} \mid \tilde{x}_{0: t+1}^{(i)}, z_{t}^{(i, j)}, y_{0: t}\right)$. If (37) is chosen as the proposal function, i.e., $\pi\left(z_{t+1} \mid \tilde{x}_{0: t+1}^{(i)}, y_{0: t}\right)=$ $p_{N_{z}}\left(z_{t+1} \mid \tilde{x}_{0: t+1}^{(i)}, y_{0: t}\right)$ in the marginal PF, then sampling from (38) would be exactly the same as what we did. In addition, like the marginal $\mathrm{PF}$, for each $i=1, \ldots, N_{x}$, the computational cost of $\tilde{r}_{t}^{(i, j)}, j=1, \ldots, N_{z}$, is $\mathcal{O}\left(N_{z}^{2}\right)$. However, this should not be a problem as a small $N_{z}$ of particles are usually used to approximate $p\left(z_{t} \mid x_{0: t}^{(i)}, y_{0: t}\right)$. Moreover, a couple of methods have been given in [21] to reduce this computational cost. On the other hand, if (36) is chosen as the proposal function, then $\tilde{r}_{t}^{(i, j)}=1, i=1, \ldots, N_{x}, j=1, \ldots, N_{z}$. As a result, the resampling of $\left\{\tilde{r}_{t}^{(i, 1)}, \ldots, \tilde{r}_{t}^{\left(i, N_{z}\right)}\right\}, i=1, \ldots, N_{x}$, is not needed and the computation of (11), (23) and (26) is simplified.

Remark 3.3: If system (3) has a special structure, then the construction of $\pi\left(x_{t+1} \mid x_{0: t}^{(i)}, y_{0: t}\right)$ can become simpler. We mention two cases here. First, assume that the $x$-dynamics of system (3) is independent of $z$, then $p\left(x_{t+1} \mid x_{0: t}^{(i)}, y_{0: t}\right)=$ $p\left(x_{t+1} \mid x_{t}^{(i)}\right)$ and thus we can choose $\pi\left(x_{t+1} \mid x_{0: t}^{(i)}, y_{0: t}\right)=$ $p\left(x_{t+1} \mid x_{t}^{(i)}\right)$. Systems with this special structure have been studied in the literature before, see e.g., [11]. Second, assume that system (3) takes the following form

$$
\begin{aligned}
x_{t+1} & =f_{t}^{x}\left(x_{t}, z_{t}\right)+g_{t}^{x}\left(x_{t}\right) v_{t}^{x} \\
z_{t+1} & =f_{t}^{z}\left(x_{t}, z_{t}\right)+g_{t}^{z}\left(x_{t}, z_{t}\right) v_{t}^{z} \\
y_{t} & =h_{t}\left(x_{t}, z_{t}, e_{t}\right)
\end{aligned}
$$

where $f_{t}^{x}(\cdot), f_{t}^{z}(\cdot), g_{t}^{x}(\cdot), g_{t}^{z}(\cdot)$ and $h_{t}(\cdot)$ are known functions, $v_{t}$ is assumed white and Gaussian distributed according to

$$
v_{t}=\left[\begin{array}{c}
v_{t}^{x} \\
v_{t}^{z}
\end{array}\right] \sim \mathcal{N}\left(0,\left[\begin{array}{cc}
Q_{t}^{x} & \left(Q_{t}^{z x}\right)^{T} \\
Q_{t}^{z x} & Q_{t}^{z}
\end{array}\right]\right)
$$

Then from (34), a further simplified approximation of $p\left(x_{t+1} \mid x_{0: t}^{(i)}, y_{0: t}\right) \quad$ can be obtained as $\mathcal{N}\left(\bar{x}_{t+1}^{(i)}, \Sigma_{t+1}^{(i)}+\right.$ $\left.g_{t}^{x}\left(x_{t}^{(i)}\right) Q_{t}^{x}\left(g_{t}^{x}\left(x_{t}^{(i)}\right)\right)^{T}\right)$ with $\bar{x}_{t+1}^{(i)}$ and $\Sigma_{t+1}^{(i)}$, respectively, the mean and the covariance of the discrete distribution $\left\{\tilde{x}_{t+1}^{(i, j)}, j=1, \ldots, N_{z}\right\}$ with probability mass $\bar{q}_{t}^{(i, j)}$ associated with the element $\tilde{x}_{t+1}^{(i, j)} \sim p\left(x_{t+1} \mid x_{t}^{(i)}, \bar{z}_{t}^{(i, j)}\right)$. For this special case, $\pi\left(x_{t+1} \mid x_{0: t}^{(i)}, y_{0: t}\right)=\mathcal{N}\left(\bar{x}_{t+1}^{(i)}, \Sigma_{t+1}^{(i)}+\right.$ $\left.g_{t}^{x}\left(x_{t}^{(i)}\right) Q_{t}^{x}\left(g_{t}^{x}\left(x_{t}^{(i)}\right)\right)^{T}\right)$.

While we have assumed the proposal functions in the form of $\pi\left(x_{t} \mid x_{0: t-1}^{(i)}, y_{0: t-1}\right)$ and $\pi\left(z_{t} \mid \tilde{x}_{0: t}^{(i)}, y_{0: t-1}\right)$, it is possible to choose proposal functions in more general forms. For example, in the importance sampling of (24), the proposal function $\pi\left(z_{t} \mid x_{0: t}^{(i)}, y_{0: t-1}\right)$ can be replaced by another proposal function in the form of $\pi\left(z_{t} \mid \tilde{x}_{0: t+1}^{(i)}, y_{0: t-1}\right)$. Moreover, this new proposal function, together with the two proposal functions $\pi\left(x_{t} \mid x_{0: t-1}^{(i)}, y_{0: t-1}\right)$ and $\pi\left(z_{t} \mid \tilde{x}_{0: t}^{(i)}, y_{0: t-1}\right)$ can be further made dependent on $y_{t}$. That is, in the importance sampling of (14), (21) and (24), the proposal functions in the form of $\pi\left(x_{t} \mid x_{0: t-1}^{(i)}, y_{0: t}\right), \pi\left(z_{t} \mid x_{0: t}^{(i)}, y_{0: t}\right)$ and $\pi\left(z_{t} \mid \tilde{x}_{0: t+1}^{(i)}, y_{0: t}\right)$ can be used, respectively. Due to the space limitation, we do not discuss this issue here and we refer the interested reader to, for example, [2, 7, 13, 23] for relevant discussions. Finally, we remaind that if these proposal functions in more general forms are used, then slight modification is needed to make to the DPF algorithm.

3) Computing the state estimate: A common application of a PF is to compute the state estimate, i.e., the expected mean of the state. For system (3), the state estimate of $x_{t}$ and $z_{t}$ are defined as

$$
\bar{x}_{t}=\mathrm{E}_{p\left(x_{t} \mid y_{0: t}\right)}\left(x_{t}\right), \bar{z}_{t}=\mathrm{E}_{p\left(z_{t} \mid y_{0: t}\right)}\left(z_{t}\right)
$$

Then the approximation of $\bar{x}_{t}$ and $\bar{z}_{t}$ can be computed in the following way for the DPF. Note from (17) that $p\left(x_{t} \mid y_{0: t}\right)$ has an empirical approximation $p_{N_{x}}\left(x_{t} \mid y_{0: t}\right)=\sum_{i=1}^{N_{x}} w_{t}^{(i)} \delta\left(x_{t}-\right.$ $\left.\tilde{x}_{t}^{(i)}\right)$. Then, an approximation $\hat{x}_{t}$ of $\bar{x}_{t}$ can be calculated in the following way

$$
\hat{x}_{t}=\mathrm{E}_{p_{N_{x}}\left(x_{t} \mid y_{0: t}\right)}\left(x_{t}\right)=\sum_{i=1}^{N_{x}} w_{t}^{(i)} \tilde{x}_{t}^{(i)}
$$

Analogously, note from (20) and (22) that $p\left(z_{t} \mid y_{0: t}\right)$ has an empirical approximation $p_{N_{x}, N_{z}}\left(z_{t} \mid y_{0: t}\right)=$ $\frac{1}{N_{x}} \sum_{i=1}^{N_{x}} \sum_{j=1}^{N_{z}} \bar{q}_{t}^{(i, j)} \delta\left(z_{t}-\bar{z}_{t}^{(i, j)}\right)$. Then, an approximation $\hat{z}_{t}$ of $\bar{z}_{t}$ can be calculated as

$$
\hat{z}_{t}=\mathrm{E}_{p_{N_{x}, N_{z}}\left(z_{t} \mid y_{0: t}\right)}\left(z_{t}\right)=\frac{1}{N_{x}} \sum_{i=1}^{N_{x}} \sum_{j=1}^{N_{z}} \bar{q}_{t}^{(i, j)} \bar{z}_{t}^{(i, j)}
$$

\section{Discussion}

In the DPF algorithm, the summation calculation in the normalizing factor (the denominator) of $w_{t}^{(i)}$ in (18) and the first resampling, i.e., the resampling of $\left\{\tilde{x}_{t}^{(i)}, \tilde{z}_{t}^{(i, 1)}, \tilde{r}_{t}^{(i, 1)}, \ldots, \tilde{z}_{t}^{\left(i, N_{z}\right)}, \tilde{r}_{t}^{\left(i, N_{z}\right)}\right\}, i=1, \ldots, N_{x}$, are the only operations that cannot be implemented in parallel. Besides, the remaining operations including the second resampling, i.e., the resampling of the particles $\bar{z}_{t}^{(i, j)}, i=1, \ldots, N_{x}$, $j=1, \ldots, N_{z}$, can be divided into $N_{x}$ independent parts in terms of the index $i$ and can thus be implemented in parallel. In this sense, we say that the DPF has the advantage over the 


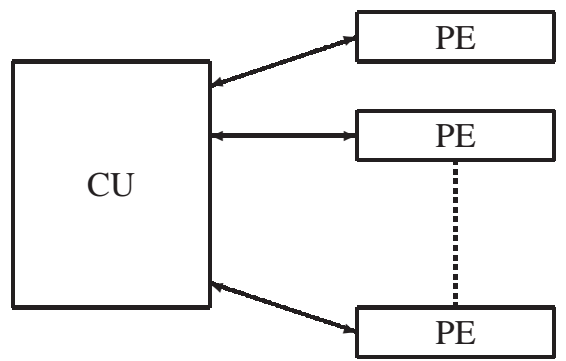

Fig. 3. The architecture of the PF with parallel structure. It consists of a central unit $(\mathrm{CU})$ and a number of processing elements (PE) where the $\mathrm{CU}$ handles the operations that cannot be implemented in parallel and the PEs are run in parallel to deal with the operations that can be implemented in parallel.

regular PF in that the DPF can increase the level of parallelism of the PF.

The underlying idea of the DPF is different from that of the distributed PFs, such as the DRPA-PF and the DRNAPF (see Section I), while they all have parallel structure. This difference results in a couple of unique features of the DPF in contrast with the distributed PFs, which identify the potential of the DPF in the application of PFs in real-time systems and the parallel implementation of PFs. In the remaining part of this section, we first briefly review the DRPA-PF and the DRNA-PF, and then we point out the unique features of the DPF. Finally, we show that there exist two ways to further increase the level of parallelism of the DPF.

Before the discussion, it should be noted that all the DPF, the DRPA-PF and the DRNA-PF have the architecture as shown in Fig. 3.

\section{A. Review of the DRPA-PF and the DRNA-PF [5]}

Assume that the sample space contains $M$ particles, where $M$ is assumed to be the number of particles that is needed for the sampling importance resampling PF (SIR-PF) [13] or the bootstrap PF [15] to achieve a satisfactory performance for the filtering problem of system (1). Then the sample space is divided into $K$ disjoint strata where $K$ is an integer and satisfies $1 \leq K \leq M$. Further assume that each stratum corresponds to a PE. Before resampling each PE thus has $N$ particles where $N=M / K$ is an integer.

The sequence of operations performed by the $k$ th PE and the CU for the DRPA-PF is shown in Fig. 4. The inter-resampling is performed on the $\mathrm{CU}$ and its function is to calculate the number of particles $N^{(k)}$ that will be produced after resampling for the $k$ th PE. $E\left(N^{(k)}\right)$ should be proportional to the weight $W^{(k)}$ of the $k$ th PE which is defined as the sum of the weights of the particles inside the $k$ th PE. In particular, $N^{(k)}$ is calculated using the residual systematic resampling (RSR) algorithm proposed in [4]. Once $N^{(k)}, k=1, \ldots, K$, are known, resampling is performed inside the $K$ PEs independently which is referred to as the intra-resampling. Note that after resampling, for $k=1, \ldots, K$, the $k$ th PE has $N^{(k)}$ particles and $N^{(k)}$ is a random number because it depends on the overall distribution of the weights $W^{(k)}, k=1, \ldots, K$. On the other hand, note that each PE is supposed to be responsible for processing $N$ particles and to perform the same operations in time. Therefore after resampling, the exchange of particles
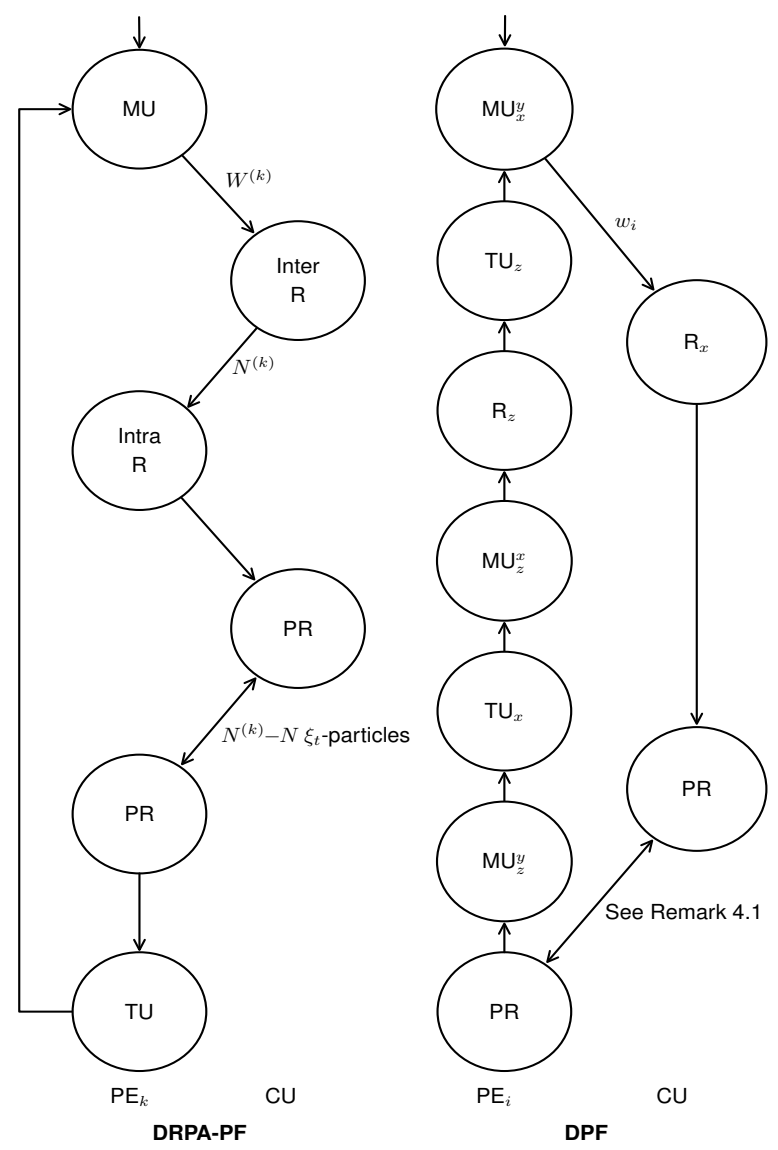

Fig. 4. Sequence of operations performed by the specified PE and the CU for the DRPA-PF and the DPF. The data transmitted between the specified $\mathrm{PE}$ and the $\mathrm{CU}$ and between different PEs are marked. For the DRPA$\mathrm{PF}$, the abbreviations are MU (measurement update of $\xi_{0: t}$ based on $y_{t}$ ), Inter $\mathrm{R}$ (inter resampling), Intra $\mathrm{R}$ (intra resampling), PR (particle routing), and TU (generation of particles $\xi_{t+1}^{(l)}, l=1, \ldots, M$ ). For the DPF, the abbreviations are $\mathrm{MU}_{x}^{y}$ (measurement update of $x_{0: t}$ based on $\left.y_{t}\right), \mathbf{R}_{x}$ (resampling of $\left.\left\{\tilde{x}_{t}^{(i)}, \tilde{z}_{t}^{(i, 1)}, \tilde{r}_{t}^{(i, 1)}, \ldots, \tilde{z}_{t}^{\left(i, N_{z}\right)}, \tilde{r}_{t}^{\left(i, N_{z}\right)}\right\}, i=1, \ldots, N_{x}\right)$, $\mathrm{PR}$ (particle routing), $\mathrm{MU}_{z}^{y}$ (measurement update of $z_{t}$ based on $y_{t}$ ), $\mathrm{TU}_{x}$ (generation of particles $\left.\tilde{x}_{t+1}^{(i)}, i=1, \ldots, N_{x}\right), \mathrm{MU}_{z}^{x}$ (measurement update of $z_{t}$ based on $x_{t+1}$ ), $\mathrm{R}_{z}$ (resampling of $\bar{z}_{t}^{(i, j)}, i=1, \ldots, N_{x}, j=1, \ldots, N_{z}$ ) and $\mathrm{TU}_{z}$ (generation of particles $\tilde{z}_{t+1}^{(i, j)}, i=1, \ldots, N_{x}, j=1, \ldots, N_{z}$ ).

among the PEs has to be performed such that each PE has $N$ particles. This procedure is referred as particle routing and is conducted by the CU. According to [5], the DRPA$\mathrm{PF}$ requires a complicated scheme for particle routing due to the proportional allocation rule. In order to shorten the delay caused by the complicated particle routing scheme in the DRPA-PF, the DRNA is in turn proposed in [5].

\section{B. Unique features of the DPF}

The parallel structure of the DPF is created by decomposing the state space, differing from the parallel structure of the distributed PFs which is created by dividing the sample space. In the following, we will show that this difference results in a couple of unique features of the DPF.

Before the discussion, the sequence of operations performed by the $i$ th PE and the CU for the DPF is shown in Fig. 4. The summation calculation in the normalizing factor of $w_{t}^{(i)}$ in (18) and the resampling of $\left\{\tilde{x}_{t}^{(i)}, \tilde{z}_{t}^{(i, 1)}, \tilde{r}_{t}^{(i, 1)}, \ldots, \tilde{z}_{t}^{\left(i, N_{z}\right)}, \tilde{r}_{t}^{\left(i, N_{z}\right)}\right\}$, 
$i=1, \ldots, N_{x}$, are performed on the CU. Assume that there are $N_{x}$ PEs, and for each $i=1, \ldots, N_{x}$, the $i$ th PE handles the $i$ th independent part of the remaining operations of the DPF. In particular, for each $i=1, \ldots, N_{x}$, the $i$ th PE handles the resampling of the particles $\bar{z}_{t}^{(i, j)}, j=1, \ldots, N_{z}$. Therefore, the resampling of the particles $\bar{z}_{t}^{(i, j)}, i=1, \ldots, N_{x}, j=1, \ldots, N_{z}$, is run in parallel on the PEs.

Remark 4.1: In the particle routing of the DPF, the data transmitted between different PEs depends on the resampling result of $\left\{\tilde{x}_{t}^{(i)}, \tilde{z}_{t}^{(i, 1)}, \tilde{r}_{t}^{(i, 1)}, \ldots, \tilde{z}_{t}^{\left(i, N_{z}\right)}, \tilde{r}_{t}^{\left(i, N_{z}\right)}\right\}, i=1, \ldots, N_{x}$. More specifically, there will be no data transmitted through the $i$ th PE if $\left\{\tilde{x}_{t}^{(i)}, \tilde{z}_{t}^{(i, 1)}, \tilde{r}_{t}^{(i, 1)}, \ldots, \tilde{z}_{t}^{\left(i, N_{z}\right)}, \tilde{r}_{t}^{\left(i, N_{z}\right)}\right\}$ is selected only once in the resampling. Otherwise, the data transmitted through the $i$ th PE will be $\left\{\tilde{x}_{t}^{(m)}, \tilde{z}_{t}^{(m, 1)}, \tilde{r}_{t}^{(m, 1)}, \ldots, \tilde{z}_{t}^{\left(m, N_{z}\right)}\right.$, $\left.\tilde{r}_{t}^{\left(m, N_{z}\right)}\right\}$ for some $m=1, \ldots, N_{x}$. In particular, if $\left\{\tilde{x}_{t}^{(i)}, \tilde{z}_{t}^{(i, 1)}, \tilde{r}_{t}^{(i, 1)}, \ldots, \tilde{z}_{t}^{\left(i, N_{z}\right)}, \tilde{r}_{t}^{\left(i, N_{z}\right)}\right\}$ is selected more than once, then $m=i$; if $\left\{\tilde{x}_{t}^{(i)}, \tilde{z}_{t}^{(i, 1)}, \tilde{r}_{t}^{(i, 1)}, \ldots, \tilde{z}_{t}^{\left(i, N_{z}\right)}, \tilde{r}_{t}^{\left(i, N_{z}\right)}\right\}$ is not selected, then $m=1, \ldots, N_{x}$ and $m \neq i$. Therefore, the data transmitted between any two PEs, say, the $i_{1}$ th $\mathrm{PE}$ and the $i_{2}$ th $\mathrm{PE}$, will be either zero or $\left\{\tilde{x}_{t}^{(m)}, \tilde{z}_{t}^{(m, 1)}, \tilde{r}_{t}^{(m, 1)}, \ldots, \tilde{z}_{t}^{\left(m, N_{z}\right)}, \tilde{r}_{t}^{\left(m, N_{z}\right)}\right\}$ for either $m=i_{1}$ or $m=i_{2}$.

In contrast to the DRPA-PF, the DPF allows a simpler particle routing scheme. For the DRPA-PF, since after resampling the $k$ th PE has $N^{(k)}$ particles that is a random number, a complicate scheme has to be used for the DRPA-PF to make all $K$ PEs has equally $N$ particles. For the DPF, however, since after the resampling of $\left\{\tilde{x}_{t}^{(i)}, \tilde{z}_{t}^{(i, 1)}, \tilde{r}_{t}^{(i, 1)}, \ldots, \tilde{z}_{t}^{\left(i, N_{z}\right)}, \tilde{r}_{t}^{\left(i, N_{z}\right)}\right\}$, $i=1, \ldots, N_{x}$, all $N_{x}$ PEs still have the same number of particles, then the DPF allows a simpler particle routing scheme and actually each PE can be treated as a single particle in the particle routing.

Given a PF with parallel structure, it works most efficiently if each PE handles the same number of particles. The efficiency of the DRPA-PF usually decreases, since the numbers of particles produced by each PE are not evenly but randomly distributed among the PEs. To be specific, note that the time used by the $k$ th PE to produce $N^{(k)}$ particles, $k=1, \ldots, K$, after resampling are usually not the same. This observation implies that the time used by the DRPA to produce the particles after resampling is determined by the $k^{*}$ th PE that produces the largest $N^{\left(k^{*}\right)}$. Clearly, the more unevenly the numbers of particles produced by each PE are distributed, the more time the DRPA takes to produce the particles after resampling. Especially, in the extreme case that $N^{\left(k^{*}\right)} \gg N^{(k)}$ with $k=1, \ldots, K$, and $k \neq k^{*}$, the efficiency of the DRPA$\mathrm{PF}$ will be decreased significantly. However, for the DPF, the $i$ th PE that handles the resampling of particles $\bar{z}_{t}^{(i, j)}$, $j=1, \ldots, N_{z}$, produces, after resampling, the same number of particles $z_{t}^{(i, j)}, j=1, \ldots, N_{z}$. Therefore, the DPF does not have the efficiency decrease problem of the DRPA-PF.

Besides, it will be verified by two numerical examples in the subsequent section that, the DPF has the potential to achieve in a shorter execution time the same level of performance as the bootstrap PF. However, the DRNA-PF actually trades PF performance for speed improvement $[5,22]$.

Remark 4.2: The ideal minimum execution time $T_{\mathrm{ex}}$ of the DRPA-PF and the DRNA-PF have been given in $[5,22]$. Analogously, we can also give the ideal minimum execution time of the DPF. Like [5,22], the following assumptions are made. Consider an implementation with a pipelined processor. Assume that the execution time of the particle generation and the importance weights calculation of every particle is $L T_{\text {clk }}$ where $L$ is the latency due to the pipelining and $T_{\text {clk }}$ is the clock period. Also assume that the resampling takes the same amount of time as the particle generation and the importance weights calculation. As a result, we have the ideal minimum execution time of the DPF as $T_{\mathrm{ex}}^{\mathrm{DPF}}=\left(2 N_{z}+\right.$ $\left.L+N_{x}+M_{r}+1\right) T_{\text {clk }}$. Here, $2 N_{z}$ represents the delay due to the resampling of the particles $\bar{z}_{t}^{(i, j)}, j=1, \ldots, N_{z}$, and the corresponding particle generation and importance weights calculation, $N_{x}$ represents the delay due to the resampling of $\left\{\tilde{x}_{t}^{(i)}, \tilde{z}_{t}^{(i, 1)}, \tilde{r}_{t}^{(i, 1)}, \ldots, \tilde{z}_{t}^{\left(i, N_{z}\right)}, \tilde{r}_{t}^{\left(i, N_{z}\right)}\right\}, i=1, \ldots, N_{x}, M_{r}$ is the delay due to the particle routing and the extra one $T_{\text {clk }}$ is due to the particle generation and importance weight calculation of the particle $x_{t}^{(i)}$.

\section{Two ways to further increase the level of parallelism of the $D P F$}

The first resampling of the DPF, i.e., resampling of $\left\{\tilde{x}_{t}^{(i)}, \tilde{z}_{t}^{(i, 1)}, \tilde{r}_{t}^{(i, 1)}, \ldots, \tilde{z}_{t}^{\left(i, N_{z}\right)}, \tilde{r}_{t}^{\left(i, N_{z}\right)}\right\}, i=1, \ldots, N_{x}$, is the major operation that cannot be implemented in parallel. If $N_{x}$ is large, then this resampling will cause a large delay. In order to further increase the level of parallelism of the DPF and shorten the execution time, it is valuable to find ways to handle this problem.

Two possible ways will be given here. The first one is straightforward and is to employ any of the distributed resampling algorithms proposed in $[5,22]$ to perform the first resampling of the DPF and besides, the remaining parts of the DPF stay unchanged. Nonetheless, we prefer the DRPA to the other distributed resampling algorithms, since it can produce the same result as the systematic resampling [20] according to [4].

Compared to the first way, the second way only applies to high dimensional system (1) and it is based on an extension of the DPF. We have assumed above that the state $\xi_{t}$ is decomposed into two parts according to (2). Actually, the DPF can be extended to handle the case where the state $\xi_{t}$ is decomposed into more than two (at most $n_{\xi}$ ) parts. For illustration, we briefly consider the case where the state $\xi_{t}$ is decomposed into three parts. The more general case can be studied using the induction principle.

For convenience, assume that the state $x_{t}$ in (2) can be further decomposed into two parts, i.e.,

$$
x_{t}=\left[\begin{array}{l}
x_{1, t} \\
x_{2, t}
\end{array}\right]
$$

and accordingly, system (3) can be further decomposed into the following form

$$
\begin{aligned}
x_{1, t+1} & =f_{t}^{x_{1}}\left(x_{1, t}, x_{2, t}, z_{t}, v_{t}^{x_{1}}\right) \\
x_{2, t+1} & =f_{t}^{x_{2}}\left(x_{1, t}, x_{2, t}, z_{t}, v_{t}^{x_{2}}\right) \\
z_{t+1} & =f_{t}^{z}\left(x_{1, t}, x_{2, t}, z_{t}, v_{t}^{z}\right) \\
y_{t} & =h_{t}\left(x_{1, t}, x_{2, t}, z_{t}, e_{t}\right)
\end{aligned}
$$




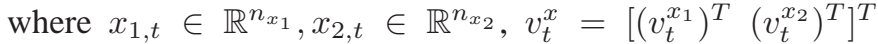
with $v_{t}^{x_{1}} \in \mathbb{R}^{n_{v^{x_{1}}}}, v_{t}^{x_{2}} \in \mathbb{R}^{n_{v^{x_{2}}}}$. Assume that the probability densities $p\left(x_{1,0} \mid y_{-1}\right)=p\left(x_{1,0}\right), p\left(x_{2,0} \mid x_{1,0}, y_{-1}\right)=$ $p\left(x_{2,0} \mid x_{1,0}\right), p\left(z_{0} \mid x_{1,0}, x_{2,0}, y_{-1}\right)=p\left(z_{0} \mid x_{1,0}, x_{2,0}\right)$ and for $t \geq 0, p\left(x_{1, t+1} \mid x_{1, t}, x_{2, t}, z_{t}\right), p\left(x_{2, t+1} \mid x_{1, t: t+1}, x_{2, t}, z_{t}\right)$, $p\left(z_{t} \mid x_{1, t: t+1}, x_{2, t: t+1}, z_{t}\right)$ and $p\left(y_{t} \mid x_{1, t}, x_{2, t}, z_{t}\right)$ are known.

The filtering problem of system (45) can be split into three nested sub-problems according to the following factorization

$$
\begin{gathered}
p\left(z_{t}, x_{1,0: t}, x_{2,0: t} \mid y_{0: t}\right)=p\left(z_{t} \mid x_{1,0: t}, x_{2,0: t}, y_{0: t}\right) \\
\quad \times p\left(x_{2,0: t} \mid x_{1,0: t}, y_{0: t}\right) p\left(x_{1,0: t} \mid y_{0: t}\right)
\end{gathered}
$$

where for $i=1,2, x_{i, 0: t} \triangleq\left\{x_{i, 0}, \ldots, x_{i, t}\right\}$. It can be shown that the DPF can be extended to handle the filtering problem of system (45) by using PFs to solve the three nested subproblems. Roughly speaking, a PF with $N_{x_{1}}$ particles $\left(x_{1,0: t}^{(i)}\right.$, $\left.i=1, \ldots, N_{x_{1}}\right)$ will be used to estimate $p\left(x_{1,0: t} \mid y_{0: t}\right)$, and for each $i=1, \ldots, N_{x_{1}}$, a PF with $N_{x_{2}}$ particles $\left(x_{2,0: t}^{(i, j)}, j=\right.$ $\left.1, \ldots, N_{x_{2}}\right)$ will be used to estimate $p\left(x_{2,0: t} \mid x_{1,0: t}^{(i)}, y_{0: t}\right)$, and for each $i=1, \ldots, N_{x_{1}}$ and $j=1, \ldots, N_{x_{2}}$, a PF with $N_{z}$ particles will be used to estimate $p\left(z_{t} \mid x_{1,0: t}^{(i)}, x_{2,0: t}^{(i, j)}, y_{0: t}\right)$.

Similar to the DPF based on (4), the major operation that cannot be implemented in parallel in the DPF based on (46) is its first resampling, i.e., the resampling of $N_{x_{1}}$ composite particles. If a satisfactory performance of the DPF based on (46) can be achieved with $N_{x_{1}}+N_{x_{2}} \ll N_{x}$, then the number of composite particles involved in the first resampling of the DPF will be reduced from $N_{x}$ to $N_{x_{1}}$. Therefore, in this way the level of parallelism of the DPF is further increased. If the DPF is implemented in parallel, then the execution time of the DPF will be further decreased as well. However, it should be noted that $N_{x_{1}} \cdot N_{x_{2}}$ PEs are required to fully exploit the parallelism of the DPF based on (46). Due to the space limitation, we cannot include the extension of the DPF in this paper and instead we refer the reader to [10] for the details.

\section{NUMERICAL EXAMPLES}

In this section we will test how the DPF performs on two examples. The simulations are performed using Matlab under the Linux operating system. The platform is a server consisting of eight Intel(R) Quad Xeon(R) CPUs (2.53GHz).

\section{A. Algorithms tested}

For the two examples, the bootstrap PF is implemented in the standard fashion, using different number of particles $(M)$. The DPF is implemented according to Section III-B for different combinations of " $x$ and $z$ particles" $\left(N_{x}\right.$ and $N_{z}$ ). The DRPA-PF according to [5] is tested as well, using different number of PEs $(K)$. The formulas of [5] has been closely followed, but the implementation is our own, and it is of course possible that it can be further trimmed. In addition, as suggested in $[17,20]$ systematic resampling is chosen as the resampling algorithm for all algorithms tested.

\section{B. Performance evaluation: Accuracy}

In the tests, the performance of all algorithms are evaluated by 20000 Monte Carlo simulations. Basically, the accuracy of the state estimate is measured by the Root Mean Square Error (RMSE) between the true state and the state estimate. For example, the RMSE of $\hat{x}$ is defined as

$$
\text { RMSE of } \hat{x}=\sqrt{\frac{1}{250} \sum_{t=1}^{250} \frac{1}{20000} \sum_{i=1}^{20000}\left\|x_{t}^{i}-\hat{x}_{t}^{i}\right\|^{2}}
$$

where with a slight abuse of notation, $x_{t}^{i}$ denotes the true state at time $t$ for the $i$ th simulation and $\hat{x}_{t}^{i}$ is the corresponding state estimate. It is also tested how well the RMSE reflects the accuracy of the estimated posterior densities (See Remark 5.1 for more details).

\section{Performance evaluation: Timing}

One objective with the simulations is to assess the potential efficiency of the parallel implementation of the DPF. For that purpose, we record the following times

- $T_{\mathrm{si}}$ : This is the average execution time of the sequential implementation of a PF.

- $T_{\mathrm{cp}}$ : This is the average time used by the operations that cannot be implemented in parallel in a PF.

- $T_{\mathrm{pi}}$ : This is the potential execution time of parallel implementation of a PF. For the bootstrap PF with centralized resampling and the DPF, it is calculated according to $T_{\mathrm{pi}}=T_{\mathrm{cp}}+\left(T_{\mathrm{si}}-T_{\mathrm{cp}}\right) / N_{\mathrm{PE}}$ where $N_{\mathrm{PE}}$ is the number of processing elements. For the DPF, let $N_{\mathrm{PE}}=N_{x}$. For the bootstrap PF with centralized resampling, let $N_{\mathrm{PE}}$ be the maximal $N_{x}$ in the simulation of the corresponding example. Here, the bootstrap PF with centralized resampling means that besides the resampling, the remaining particle generation and importance weights calculation of the bootstrap PF are implemented in parallel. For the DRPA-PF, $T_{\mathrm{pi}}$ is calculated according to $T_{\mathrm{pi}}=T_{\mathrm{cp}}+T_{\mathrm{mir}}+$ $\left(T_{\mathrm{si}}-T_{\mathrm{cp}}-T_{\mathrm{mir}}\right) / N_{\mathrm{PE}}$ where $N_{\mathrm{PE}}=K$ and $T_{\mathrm{mir}}$ is the average maximal intra-resampling time for the DRPA-PF.

\section{Performance evaluation: Divergence failures}

The rate $r_{d}$ is used to reveal how often a PF diverges in the 20000 Monte Carlo simulations. The bootstrap PF and the DRPA-PF are said to diverge if their importance weights are all equal to zero in the simulation. The DPF is said to diverge if $w_{t}^{(i)}, i=1, \ldots, N_{x}$, are all equal to zero in the simulation. Once the divergence of a PF is detected, the PF will be rerun.

\section{E. Sketch of the simulation}

For the two examples, the bootstrap PF using $M$ particles is first implemented and its accuracy measured by the RMSE will be treated as the reference level. Then it is shown that the DPF using suitable $N_{x}$ and $N_{z}$ " $x$ and $z$ particles" can achieve the same level of accuracy. In turn, the DRPA-PF using $M$ particles, but with different number of processing elements is also implemented. Finally, the bootstrap PF using $2 M$ particles is implemented. 
TABLE I

Simulation Result For System (48) With (49) - "SEe SECtions V-B - V-D for EXPlanations of the NUmbers"

\begin{tabular}{cccccc}
\hline \hline Case & RMSE of $\left[\hat{x}_{t}, \hat{z}_{t}\right]$ & $T_{\mathrm{si}}(\mathrm{Sec})$ & $T_{\mathrm{cp}}(\mathrm{Sec})$ & $T_{\mathrm{pi}}(\mathrm{Sec})$ & $r_{\mathrm{d}}$ \\
\hline Bootstrap PF, $M=1000$ & {$[2.0173,2.3322]$} & $\mathbf{0 . 1 8 9 1}$ & 0.0313 & 0.0326 & 0.0155 \\
DPF, $N_{x}=100, N_{z}=19$ & {$[2.0104,2.3497]$} & 0.3545 & $\mathbf{0 . 0 1 6 8}$ & $\mathbf{0 . 0 2 0 2}$ & 0.0133 \\
DPF, $N_{x}=120, N_{z}=19$ & {$[1.9914,2.3045]$} & 0.3901 & 0.0176 & 0.0207 & 0.0175 \\
DPF, $N_{x}=110, N_{z}=24$ & {$[1.9907,2.3154]$} & 0.4127 & 0.0175 & 0.0211 & 0.0113 \\
DPF, $N_{x}=120, N_{z}=24$ & {$[1.9906,2.3259]$} & 0.4338 & 0.0179 & 0.0214 & 0.0076 \\
DRPA-PF, $M=1000, K=40$ & {$[2.0222,2.3557]$} & 0.6324 & 0.0671 & 0.0878 & 0.0124 \\
DRPA-PF, $M=1000, K=25$ & {$[2.0332,2.4049]$} & 0.4769 & 0.0565 & 0.0799 & 0.0124 \\
\hline Bootstrap PF, $M=2000$ & {$[\mathbf{1 . 9 7 1 4}, \mathbf{2 . 2 6 6 4}]$} & 0.2579 & 0.0510 & 0.0528 & $\mathbf{0 . 0 0 5 9}$ \\
\hline
\end{tabular}

\section{F. Two dimensional example}

Consider the following two dimensional nonlinear system

$$
\begin{aligned}
x_{t+1} & =x_{t}+\frac{z_{t}}{1+z_{t}^{2}}+v_{t}^{x} \\
z_{t+1} & =x_{t}+0.5 z_{t}+\frac{25 z_{t}}{1+z_{t}^{2}}+8 \cos (1.2 t)+v_{t}^{z} \\
y_{t} & =\operatorname{atan}\left(x_{t}\right)+\frac{z_{t}^{2}}{20}+e_{t}
\end{aligned}
$$

where $\left[\begin{array}{ll}x_{0} & z_{0}\end{array}\right]^{T}$ is assumed Gaussian distributed with $\left[\begin{array}{ll}x_{0} & z_{0}\end{array}\right]^{T} \sim \mathcal{N}\left(0, I_{2 \times 2}\right), v_{t}=\left[\begin{array}{ll}v_{t}^{x} & v_{t}^{z}\end{array}\right]^{T}$ and $e_{t}$ are assumed white and Gaussian distributed with

$$
v_{t} \sim \mathcal{N}\left(0,\left[\begin{array}{cc}
1 & 0.1 \\
0.1 & 10
\end{array}\right]\right), \text { and } e_{t} \sim \mathcal{N}(0,1)
$$

For the DPF, the proposal functions are chosen according to Remark 3.3 and (36). The simulation result over [1 250] is shown in Table I, from which it can be seen that the DPF has the potential to achieve in a shorter execution time the same level of accuracy as the bootstrap PF.

Remark 5.1: In Table I, the accuracy of the algorithms is measured entirely through the RMSE of the state estimate (47). Since the PF actually computes estimates of the posterior density $p\left(z_{t}, x_{0: t} \mid y_{0: t}\right)$ one may discuss if this would be a more appropriate quantity to evaluate for comparison. Actually, the state estimates $\hat{x}_{t}$ could be quite accurate even though the estimate of the posterior density is poor. To evaluate that, we computed an accurate value of the true posterior density using the bootstrap PF with "many" (100000) particles, and compared that with the estimates of the posterior densities using the bootstrap PF and the DPF with fewer $(M=1000$ and $N_{x}=100, N_{z}=19$ ) particles. To avoid smoothing issues for the empirical approximations of the posterior densities, we made the comparisons for the posterior cumulative distributions (empirical approximations of the posterior cumulative distributions). The result is shown in Fig. 5. Moreover, let $\bar{x}_{t}^{i}$ denote the true mean of $x_{t}^{i}$, then (47) with $x_{t}^{i}$ replaced by $\bar{x}_{t}^{i}$ is also calculated: it is 0.7239 for the bootstrap PF with $M=1000$, and 0.6851 for the DPF with $N_{x}=100$ and $N_{z}=19$. From the above simulation results, we see that the DPF is at least as good as the bootstrap PF in approximating the posterior cumulative distribution. We conclude that the RMSE (47) gives a fair evaluation of the accuracy of the state estimate produced by the DPF for system (48) with (49).

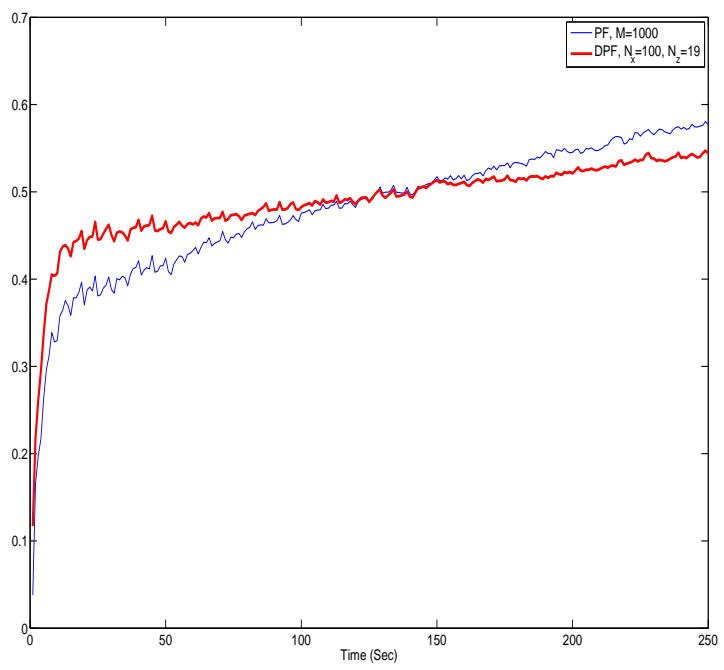

Fig. 5. The distance $\sum_{x_{t}}\left|F\left(x_{t} \mid y_{0: t}\right)-\tilde{F}\left(x_{t} \mid y_{0: t}\right)\right|$ between the "true" posterior cumulative distribution $F\left(x_{t} \mid y_{0: t}\right)$ and the empirical approximation of the posterior cumulative distribution $\tilde{F}\left(x_{t} \mid y_{0: t}\right)$ obtained by using the bootstrap PF and the DPF (with $M=1000$ and $N_{x}=100, N_{z}=19$ particles, respectively) as a function of time $t$ (Thin curve: the bootstrap PF, Thick curve: the DPF). The result is an average over 20000 simulations.

\section{G. Four dimensional example}

Consider the following four dimensional nonlinear system

$$
\begin{aligned}
x_{1, t+1}= & 0.5 x_{1, t}+8 \sin (t)+v_{t}^{x_{1}} \\
x_{2, t+1}= & 0.4 x_{1, t}+0.5 x_{2, t}+v_{t}^{x_{2}} \\
z_{1, t+1}= & z_{1, t}+\frac{z_{2, t}}{1+z_{2, t}^{2}}+v_{t}^{z_{1}} \\
z_{2, t+1}= & z_{1, t}+0.5 z_{2, t} \\
& +\frac{25 z_{2, t}}{1+z_{2, t}^{2}}+8 \cos (1.2 t)+v_{t}^{z_{2}} \\
y_{t}= & \frac{x_{1, t}+x_{2, t}}{1+x_{1, t}^{2}}+\operatorname{atan}\left(z_{1, t}\right)+\frac{z_{2, t}^{2}}{20}+e_{t}
\end{aligned}
$$

where $x_{t}=\left[\begin{array}{ll}x_{1, t} & x_{2, t}\end{array}\right]^{T}$ and $z_{t}=\left[\begin{array}{ll}z_{1, t} & z_{2, t}\end{array}\right]^{T} \cdot\left[\begin{array}{ll}x_{0}^{T} & z_{0}^{T}\end{array}\right]^{T}$ is assumed Gaussian distributed with $\left[\begin{array}{ll}x_{0}^{T} & z_{0}^{T}\end{array}\right]^{T} \sim \mathcal{N}\left(0, I_{4 \times 4}\right)$, $v_{t}=\left[\begin{array}{ll}\left(v_{t}^{x}\right)^{T} & \left(v_{t}^{z}\right)^{T}\end{array}\right]^{T}$ with $v_{t}^{x}=\left[\begin{array}{ll}v_{t}^{x_{1}} & v_{t}^{x_{2}}\end{array}\right]^{T}$ and $v_{t}^{z}=$ $\left[\begin{array}{ll}v_{t}^{z_{1}} & v_{t}^{z_{2}}\end{array}\right]^{T}$, and $e_{t}$ are assumed white and Gaussian distributed 
TABLE II

Simulation Result For System (50) With (51) - "SEe SECTiOnS V-B - V-D FOR EXPlanAtions of the NUMbers"

\begin{tabular}{cccccc}
\hline \hline Case & RMSE of $\left[\hat{x}_{1, t}, \hat{x}_{2, t}, \hat{z}_{1, t}, \hat{z}_{2, t}\right]$ & $T_{\mathrm{si}}(\mathrm{Sec})$ & $T_{\mathrm{cp}}(\mathrm{Sec})$ & $T_{\mathrm{pi}}(\mathrm{Sec})$ & $r_{\mathrm{d}}$ \\
\hline Bootstrap PF, $M=1500$ & {$[1.1566,1.3494,2.0111,2.8241]$} & $\mathbf{0 . 2 0 2 2}$ & 0.0316 & 0.0339 & 0.0072 \\
DPF, $N_{x}=50, N_{z}=29$ & {$[1.1707,1.3678,2.0485,2.9383]$} & 0.2366 & $\mathbf{0 . 0 1 4 5}$ & $\mathbf{0 . 0 1 9 0}$ & 0.0109 \\
DPF, $N_{x}=60, N_{z}=49$ & {$[1.1633,1.3569,1.9879,2.7911]$} & 0.3255 & 0.0160 & 0.0212 & 0.0039 \\
DPF, $N_{x}=75, N_{z}=39$ & {$[1.1610,1.3537, \mathbf{1 . 9 7 9 4}, \mathbf{2 . 7 5 4 7}]$} & 0.3309 & 0.0164 & 0.0206 & 0.0040 \\
DRPA-PF, $M=1500, K=30$ & {$[1.1564,1.3490,2.0028,2.7894]$} & 0.5083 & 0.0470 & 0.0669 & 0.0084 \\
DRPA-PF, $M=1500, K=50$ & {$[1.1566,1.3495,2.0148,2.8302]$} & 0.6951 & 0.0607 & 0.0780 & 0.0107 \\
\hline Bootstrap PF, $M=3000$ & {$[\mathbf{1 . 1 5 1 8}, \mathbf{1 . 3 4 1 9}, \mathbf{1 . 9 7 9 4}, 2.7601]$} & 0.3105 & 0.0539 & 0.0573 & $\mathbf{0 . 0 0 2 1}$ \\
\hline
\end{tabular}

with

$v_{t} \sim \mathcal{N}\left(0,\left[\begin{array}{cccc}1 & 0 & 0 & 0 \\ 0 & 1 & 0 & 0 \\ 0 & 0 & 1 & 0.1 \\ 0 & 0 & 0.1 & 10\end{array}\right]\right)$, and $e_{t} \sim \mathcal{N}(0,1)(51)$

Since the " $x$-dynamics" does not depend on $z_{t}$, we let $\pi\left(x_{t+1} \mid x_{0: t}^{(i)}, y_{0: t}\right)=p\left(x_{t+1} \mid x_{t}^{(i)}\right)$ and choose the other proposal function according to (36). The simulation result over [1 150] is shown in Table II, from which it can be seen that the DPF has the potential to achieve in a shorter execution time the same level of accuracy as the bootstrap PF.

\section{H. Summary}

Regarding the accuracy, comparison of the first part of the RMSE column in Tables I and II shows that with suitably chosen $N_{x}$ and $N_{z}$, the DPF achieves the same level of accuracy as the bootstrap PF. On the other hand, with comparable number of particles (it is fair to compare $M$ with $N_{x}\left(N_{z}+1\right)$ ) the accuracy is not much worse for the DPF than for the bootstrap PF. In fact, in Table II the DPF even performs slightly better than the PF for some of the states (no statistical significance), illustrating that allocating points as in Fig. 1.c could actually be beneficial for some systems.

Regarding timing, comparison of the $T_{\mathrm{si}}$ and $T_{\mathrm{pi}}$ column in Tables I and II shows that the execution time of the DPF can be shortened significantly if the DPF can be implemented in parallel. Moreover, the DPF has a potential to offer better accuracy in shorter execution time. In particular, the $T_{\mathrm{pi}}$ of the DPF is less than that of the bootstrap PF with centralized resampling. It is valuable to note that the $T_{\mathrm{pi}}$ of the DPF is even smaller than the $T_{\mathrm{cp}}$ of the bootstrap PF with centralized resampling, which is actually the lower bound of the execution time of the bootstrap PF with centralized resampling. In addition, as discussed in Section IV-C, the $T_{\mathrm{pi}}$ of the DPF can be further shortened by using any one of the distributed resampling algorithms to perform the resampling of $\left\{\tilde{x}_{t}^{(i)}, \tilde{z}_{t}^{(i, 1)}, \tilde{r}_{t}^{(i, 1)}, \ldots, \tilde{z}_{t}^{\left(i, N_{z}\right)}, \tilde{r}_{t}^{\left(i, N_{z}\right)}\right\}, i=1, \ldots, N_{x}$. As a result, it is fair to say that the parallel implementation of the DPF has the potential to shorten the execution time of the PF.

\section{CONCLUSiOnS}

In this paper we have proposed a new way of allocating the particles in particle filtering. By first decomposing the state into two parts, the DPF splits the filtering problem into two nested sub-problems and then handles the two nested sub-problems using PFs. Returning to the questions in the intuitive preview in Section II-A, we have seen that the DPF can produce results of not much worse accuracy compared to the regular PF, with comparable number of particles. We have also seen that the structure gives a potential for more efficient calculations. The advantage of the DPF over the regular PF lies in that the DPF can increase the level of parallelism of the PF in the sense that part of the resampling has a parallel structure. The parallel structure of the DPF is created by decomposing the state space, differing from the parallel structure of the distributed PFs which is created by dividing the sample space. This difference results in a couple of unique features of the DPF in contrast with the existing distributed PFs. As a result, we believe that the DPF is a new option for parallel implementation of PFs and the application of PFs in real-time systems.

An interesting topic for future work is to study how to decompose the state given a high dimensional system such that the execution time of the parallel implementation of the DPF can be maximally reduced. Another interesting topic is to test the DPF in different types of parallel hardware, for example, graphical processing units (GPU) and field-programmable gate arrays (FPGA).

A further topic of future investigations is to generalize the line pattern in Fig. 1.c to other lines and curves that may pick up useful shapes in the posterior densities to be estimated. This essentially involves a change of state variables before the DPF is applied.

\section{REFERENCES}

[1] C. Andrieu and A. Doucet, "Particle filtering for partially observed Gaussian state space models," Journal of the Royal Statistical Society: Series B (Statistical Methodology), vol. 64, pp. 827-836, 2002.

[2] M. Arulampalam, S. Maskell, N. Gordon, and T. Clapp, "A tutorial on particle filters for online nonlinear/non-Gaussian Bayesian tracking," IEEE Transactions on Signal Processing, vol. 50, pp. 174-188, 2002.

[3] N. Bergman, "Recursive Bayesian estimation: Navigation and tracking applications," PhD thesis No 579, Linköping Studies in Science and Technology, SE-581 83 Linköping, Sweden, May 1999.

[4] M. Bolić, P. Djurić, and S. Hong, "Resampling algorithms for particle filters: A computational complexity perspective," EURASIP Journal on Applied Signal Processing, vol. 15, pp. 2267-2277, 2004.

[5] — "Resampling algorithms and architectures for distributed particle filters," IEEE Transactions on Signal Processing, vol. 53, no. 7, pp. 2442-2450, 2005.

[6] R. S. Bucy and K. D. Senne, "Digital synthesis on nonlinear filters," Automatica, vol. 7, pp. 287-298, 1971.

[7] J. Carpenter, P. Clifford, and P. Fearnhead, "Improved particle filter for nonlinear problems," IEE Proceedings - Radar, Sonar and Navigation, vol. 146 , no. 1 , pp. $2-7,1999$.

[8] G. Casella and C. P. Robert, "Rao-Blackwellisation of sampling schemes," Biometrika, vol. 83, pp. 81-94, 1996. 
[9] R. Chen and J. Liu, "Mixture Kalman filters," Journal of the Royal Statistical Society: Series B (Statistical Methodology), vol. 62, pp. 493508,2000

[10] T. Chen, T. B. Schön, H. Ohlsson, and L. Ljung, "An extension of the dencentralized particle filter with arbitrary state partitioning," Automatic Control Division, Linköping University, Tech. Rep. No. 2930, 2010.

[11] A. Doucet, N. de Freitas, K. Murphy, and S. Russell, "Rao-Blackwellised particle filtering for dynamic Bayesian networks," in Proceedings of the 16th Conference on Uncertainty in Artificial Intelligence, 2000, pp. 176183.

[12] A. Doucet, N. D. Freitas, and N. Gordonn, Sequential Monte Carlo methods in practice. New york: Springer, 2001.

[13] A. Doucet, S. Godsill, and C. Andrieu, "On sequential Monte Carlo sampling methods for Bayesian filtering," Statistics and Computing, vol. 10, pp. 197-208, 2000

[14] A. Doucet and A. M. Johansen, "A tutorial on particle filtering and smoothing: Fifteen years later," in Handbook of Nonlinear Filtering, D. Crisan and B. Rozovsky, Eds. Oxford, UK: Oxford University Press, 2009.

[15] N. Gordon, D. Salmond, and A. Smith, "Novel approach to nonlinear/non-Gaussian Bayesian state estimation," Radar and Signal Processing, IEE Proceedings F, vol. 140, no. 2, pp. 107-113, 1993.

[16] F. Gustafsson, F. Gunnarsson, N. Bergman, U. Forssell, J. Jansson, R. Karlsson, and P.-J. Nordlund, "Particle filters for positioning, navigation, and tracking," IEEE Transactions on Signal Processing, vol. 50, no. 2, pp. 425-437, 2002.

[17] J. D. Hol, T. B. Schön, and F. Gustafsson, "On resampling algorithms for particle filters," in IEEE Nonlinear Statistical Signal Processing Workshop, 2006, pp. 79-82.

[18] X. Hu, T. B. Schön, and L. Ljung, "A basic convergence result for particle filtering," IEEE Transactions on Signal Processing, vol. 56, no. 4, pp. 1337-1348, 2008.

[19] R. Kalman, "A new approach to linear filtering and prediction problems," Journal of Basic Engineering, vol. 82, no. 1, pp. 35-45, 1960.

[20] G. Kitagawa, "Monte Carlo filter and smoother for non-Gaussian nonlinear state space models," J. Comput. Graph. Statist., vol. 5, no. 1, pp. $1-25,1996$.

[21] M. Klass, N. de Freitas, and A. Doucet, "Towards practical $N^{2}$ Monte Carlo: The marginal particle filter," in Uncertainty in Artificial Intelligence, 2005.

[22] J. Míguez, "Analysis of parallelizable resampling algorithms for particle filtering," Signal Processing, vol. 87, pp. 3155-3174, 2007.

[23] M. K. Pitt and N. Shephard, "Filtering via simulation: Auxiliary particle filters," Journal of the American Statistical Association, vol. 94, no. 446, pp. 590-599, 1999.

[24] L. R. Rabiner and B. H. Juang, "An introduction to hidden Markov models," IEEE Acoust., Speech, Signal Processing Mag., pp. 4-16, 1986.

[25] T. B. Schön, F. Gustafsson, and P.-J. Nordlund, "Marginalized particle filters for mixed linear/nonlinear state-space models," IEEE Transactions on Signal Processing, vol. 53, pp. 2279-2289, 2005.

\section{ACKNOWLEDGMENT}

The authors would like to thank the reviewers for their useful comments and constructive suggestions, and the associate editor for his time handling the paper. The first author would also like to thank Umut Orguner for his continuous support and helpful discussions.

This work was supported by the Strategic Research Center MOVIII, funded by the Swedish Foundation for Strategic Research, SSF, and CADICS, a Linnaeus center funded by the Swedish Research Council.
Tianshi Chen received the M.S. degree in 2005 from Harbin Institute of Technology, and the Ph.D degree in December 2008 from Chinese University of Hong Kong. Since April 2009, he has been a postdoctoral researcher at the Division of Automatic Control, Department of Electrical Engineering, Linköping University, Linköping, Sweden. His research interests include nonlinear control theory and applications, system identification and statistical signal processing.

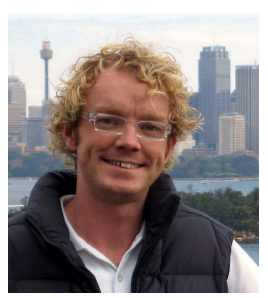

Thomas B. Schön was born in Sweden in 1977. He received the M.Sc. degree in Applied Physics and Electrical Engineering in Sep. 2001, the B.Sc. degree in Business Administration and Economics in Feb. 2001 and the Ph.D. degree in Automatic Control in Feb. 2006, all from Linköping University, Linköping, Sweden. He has held visiting positions at the University of Cambridge (UK) and the University of Newcastle (Australia). His research interests are mainly within the areas of signal processing, system identification and machine learning, with applications to the automotive and the aerospace industry. He is currently an Associate Professor at Linköping University.

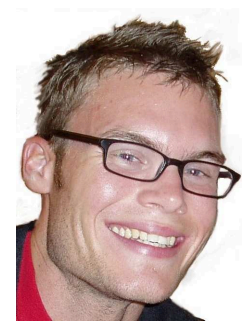

Henrik Ohlsson was born in Sweden in 1981. He received the M.Sc. degree in Applied Physics and Electrical Engineering in Oct. 2006 and his Licentiate degree in Automatic Control in Dec. 2008, all from Linköping University, Sweden. He has held visiting positions at the University of Cambridge (UK) and the University of Massachusetts (USA). His research interests are mainly within the areas of system identification and machine learning. He is currently a Ph.D. student at Linköping University.

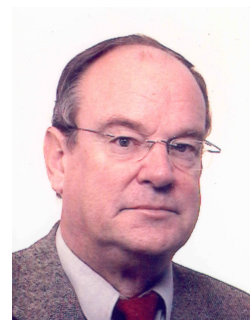

Lennart Ljung received his $\mathrm{PhD}$ in Automatic Control from Lund Institute of Technology in 1974. Since 1976 he is Professor of the chair of Automatic Control in Linköping, Sweden, and is currently Director of the Stratetic Research Center "Modeling, Visualization and Information Integration" (MOVIII). He has held visiting positions at Stanford and MIT and has written several books on System Identification and Estimation. He is an IEEE Fellow, an IFAC Fellow and an IFAC Advisor as well as a member of the Royal Swedish Academy of Sciences (KVA), a member of the Royal Swedish Academy of Engineering Sciences (IVA), an Honorary Member of the Hungarian Academy of Engineering and a Foreign Associate of the US National Academy of Engineering (NAE). He has received honorary doctorates from the Baltic State Technical University in St Petersburg, from Uppsala University, Sweden, from the Technical University of Troyes, France, from the Catholic University of Leuven, Belgium and from Helsinki University of Technology, Finland. In 2002 he received the Quazza Medal from IFAC, in 2003 he recieved the Hendrik W. Bode Lecture Prize from the IEEE Control Systems Society, and he was the recepient of the IEEE Control Systems Award for 2007. 